\title{
Non-linear MHD modeling of plasma response to Resonant Magnetic Perturbations
}

\author{
F.Orain, ${ }^{1}$ M.Bécoulet, ${ }^{1}$ G.Dif-Pradalier, ${ }^{1}$ G.Huijsmans, ${ }^{2}$ S.Pamela, ${ }^{3}$ E.Nardon, ${ }^{1}$ C.Passeron, ${ }^{1}$ G.Latu,${ }^{1}$ \\ V.Grandgirard, ${ }^{1}$ A.Fil, ${ }^{1}$ A.Ratnani, ${ }^{1}$ I.Chapman, ${ }^{4}$ A.Kirk, ${ }^{4}$ A.Thornton, ${ }^{4}$ M.Hoelzl, ${ }^{5}$ and P.Cahyna ${ }^{6}$ \\ ${ }^{1}$ CEA, IRFM, F-13108 Saint-Paul-Lez-Durance, France \\ ${ }^{2}$ ITER Organization, Route de Vinon, F-13115 Saint-Paul-Lez-Durance, France \\ ${ }^{3}$ IIFS-PIIM. Aix Marseille Université - CNRS, 13397 Marseille Cedex20, France \\ ${ }^{4}$ EURATOM/CCFE Fusion Association, Culham Science Centre, Oxon, OX14 3DB, UK \\ ${ }^{5}$ Max-Planck-Institut für Plasmaphysik, EURATOM Association, Garching, Germany \\ ${ }^{6}$ Association EURATOM/IPP.CR, Prague, Czech Republic
}

(Dated: August 11, 2013)

\begin{abstract}
The interaction of static Resonant Magnetic Perturbations (RMPs) with the plasma flows is modeled in toroidal geometry, using the non-linear resistive MHD code JOREK, which includes the $\mathrm{X}$-point and the Scrape-Off-Layer. Two-fluid diamagnetic effects, the neoclassical poloidal friction and a source of toroidal rotation are introduced in the model to describe realistic plasma flows. RMP penetration is studied taking self-consistently into account the effects of these flows and the radial electric field evolution. JET-like, MAST and ITER parameters are used in modeling. For JET-like parameters, three regimes of plasma response are found depending on the plasma resistivity and the diamagnetic rotation: at high resistivity and slow rotation, the islands generated by the RMPs at the edge resonant surfaces rotate in the ion diamagnetic direction and their size oscillates. At faster rotation, the generated islands are static and are more screened by the plasma. An intermediate regime with static islands which slightly oscillate is found at lower resistivity. In ITER simulations, the RMPs generate static islands, which forms an ergodic layer at the very edge $(\psi \geq 0.96)$ characterized by lobe structures near the X-point and results in a small strike point splitting on the divertor targets. In MAST DND geometry, lobes are also found near the X-point and the 3D-deformation of the density and temperature profiles is observed.
\end{abstract}

PACS numbers: $52.65 . \mathrm{Kj}, 52.55 . \mathrm{Tn}, 52.65 .-\mathrm{y}$

\section{INTRODUCTION}

The Edge Localized Modes (ELMs) are peelingballooning instabilities which develop in H-mode tokamak plasmas. They are characterized by the release of quasi-periodical bursts of energy and particles through the external transport barrier. The large type-I ELMs would represent a particular concern for the ITER divertor if they were not controlled [1], and the application of external Resonant Magnetic Perturbations (RMPs) is one of the most promising method to control them. Indeed, the total suppression of the type-I ELMs by RMPs was demonstrated in DIII-D [2, 3] and later in ASDEX upgrade [4] and KSTAR [5]; the strong mitigation of the ELM size was also obtained in the JET [6], MAST [7] and NSTX [8] tokamaks, motivating the use of this method in ITER [2, 9]. The non-linear MHD theory and modeling made significant progress to refine the understanding of the RMP interaction with the plasma [1015]. In particular, it was demonstrated that the penetration of the RMPs in the plasma is conditioned by the rotating plasma response [11-15]. The generation of current perturbations on the rational surfaces in the plasma can prevent magnetic reconnection, leading to the screening of RMPs. However, at certain plasma parameters, RMPs can on the contrary be amplified [16]. Non-linear MHD modeling of the rotating plasma response to RMPs in cylindrical geometry [11, 12] demonstrated that the poloidal plasma rotation, including the
$\vec{E} \times \vec{B}$ and diamagnetic rotations, both perpendicular to the magnetic field, are particularly important in the pedestal region and are likely to screen the RMPs. The aim of the present work is to extend the previous RMP modeling [12] to the more realistic toroidal geometry of the tokamak, including the X-point and the Scrape-Off Layer (SOL). The reduced MHD code JOREK [17] was enhanced to take into account the physics of the flows and the RMPs and then used for simulations. The physical model used is described in section II. In sections III, IV and $\mathrm{V}$, the penetration of the RMPs in the rotating plasma is studied in JET-like, ITER and MAST geometries respectively. In section III, the JET plasma flows in the SOL and the pedestal are first described without RMPs. Three regimes of plasma response to RMPs are highlighted which depend on the plasma resistivity and on diamagnetic rotation. The screening of the RMPs on the resonant surfaces is also discussed. In section IV, the ergodization of the very egde is observed in ITER with RMPs ; lobe structures near the X-point and 3Dcorrugation are described. The screening effect of the plasma rotation on the lobe size is demonstrated. In the Double-Null MAST geometry, the edge is also shown to be ergodized by the RMPs in section $\mathrm{V}$, going along with the 3D-deformation of the density and temperature profiles. Last, conclusions are given in section VI. 


\section{PHYSICAL MODEL}

The plasma response to RMPs is studied with the non linear reduced MHD code JOREK [17] in toroidal geometry including the X-point and the Scrape-Off Layer (SOL). The four-field model [17, 18], which encompasses the continuity equation, the parallel and perpendicular projections of the momentum equation, the energy equation and Ohm's law, is used as basic set of equations. Compared to [17], two-fluid diamagnetic effects, neoclassical friction and a source of toroidal rotation corresponding to the experimental profile are introduced in the model so as to self-consistently study the interaction between plasma flows and RMPs.

The magnetic field is decomposed in toroidal and poloidal components: $\vec{B}=F_{0} \nabla \varphi+\nabla \psi \times \nabla \varphi$, where $\varphi$ is the toroidal angle, $\psi$ is the poloidal flux and $F_{0}=R_{0} B_{\varphi 0}$; $R_{0}$ is the major radius and $B_{\varphi 0}$ is the toroidal magnetic field amplitude at the magnetic axis. $F_{0}$ is supposed to be constant in time and the poloidal magnetic field is assumed to be much lower than the toroidal one.

The two-fluid diamagnetic effects are added to the initial model [17]: the diamagnetic velocity $\vec{V}_{s}^{*}=-\nabla P_{s} \times$ $\vec{B} /\left(\rho e_{s} B^{2} / m_{i}\right)$ is taken into account for each species $s$ (electrons and ions) and is of special importance in the pedestal where the pressure profile is steep. $P_{s}$ is the scalar pressure of the species $s, e_{s}$ the charge of the species, $m_{i}$ the ion mass and $\rho=m_{i} \times n$ the ion mass density, where $n=n_{e}=n_{i}$ is the particle density. The decomposition of the fluid velocity then writes:

$$
\vec{V} \approx \vec{V}_{i}=\vec{V}_{\|, i}+\vec{V}_{E}+\vec{V}_{i}^{*}
$$

where $\vec{V}_{E}=\vec{E} \times \vec{B} / B^{2}$ is the electric drift velocity.

As for the neoclassical effects, they are considered in the momentum equation, where the pressure tensor is written $\bar{P}=\bar{I} P+\bar{\Pi}_{i, n e o}+\bar{\Pi}_{i, q v}$. The cancellation of the gyroviscous tensor $\nabla \cdot \bar{\Pi}_{i, g v}$ with the time derivative of the diamagnetic velocity $d \vec{V}_{i}^{*} / d t$ [19] is used, and the divergence of the neoclassical tensor is given by the heuristic closure taken from [20]:

$$
\nabla \cdot \bar{\Pi}_{i, n e o}=\rho \mu_{i, n e o} \frac{B^{2}}{B_{\theta}^{2}}\left(V_{\theta}-V_{\theta, n e o}\right) \vec{e}_{\theta}
$$

where $\mu_{i, n e o}$ is the neoclassical friction. This formulation expresses the friction between trapped and passing particles and constrains the poloidal velocity $V_{\theta}$ to approach the neoclassical value $V_{\theta, \text { neo }}=-k_{i} \nabla T_{i} \times \vec{B} / e B^{2} \cdot \vec{e}_{\theta}$, where $k_{i}$ is the neoclassical heat diffusivity. We have used the following definition of the unit vector in the poloidal direction:

$$
\vec{e}_{\theta}=(\nabla \psi \times \nabla \varphi) \frac{R}{|\nabla \psi|}
$$

Note that this definition implies that the orthonormal basis $\left(\vec{e}_{\psi}, \vec{e}_{\theta}, \vec{e}_{\varphi}\right)=\left(\frac{\nabla \psi}{|\nabla \psi|},(\nabla \psi \times \nabla \varphi) \frac{R}{|\nabla \psi|}, R \nabla \varphi\right)$ is left handed, and the poloidal magnetic field therefore writes

$$
B_{\theta}=B \cdot \vec{e}_{\theta}=|\nabla \psi| / R
$$

In JOREK, the grid is discretized in 2D bi-cubic Bézier finite elements in the poloidal plane [17]. Each Bézier element has its local coordinates $(s, t)$ and the local system $(s, t, \varphi)$ is related to the global cylindrical $(R, Z, \varphi)$ coordinate system in which the equations are defined, using the co- and contra-variant vectors. In this paper, the $\left(\psi_{0}, \theta, \varphi\right)$ coordinate system, more convenient for physical understanding, is also used. The equilibrium flux surfaces (defined by the equilibrium poloidal flux $\psi_{0}$ ) are calculated by solving the Grad-Shafranov equation and the angle $\theta$ is obtained from:

$$
\theta= \begin{cases}\arctan \left(\left(Z-Z_{0}\right) /\left(R-R_{0}\right)\right) & \text { for } R>R_{0} \\ \left(\pi-\arctan \left|\frac{Z-Z_{0}}{R-R_{0}}\right|\right) \times \operatorname{sign}\left(Z-Z_{0}\right) & \text { otherwise }\end{cases}
$$

The following system of equations (5)-(9) is solved for the mass density $\rho$, the parallel (ion) velocity $V_{\|, i}$, the perpendicular velocity $V_{\perp}$, the temperature $T$ and the magnetic flux $\psi$, normalized in JOREK units (the normalizations are described below):

$$
\begin{gathered}
\frac{\partial \rho}{\partial t}=-\nabla(\rho \vec{V})+\nabla \cdot\left(D_{\perp} \nabla_{\perp} \rho\right)+S_{\rho} \\
\rho \frac{F_{0} \frac{\partial V_{\|, i}}{\partial t}}{R}=\vec{b} \cdot\left(-\rho(\vec{V} \cdot \nabla)\left(\vec{V}_{\|, i}+\vec{V}_{E}\right)\right)-\nabla P \\
\left.-\bar{\nabla} \cdot \bar{\Pi}_{i, n e o}\right)+\mu_{\|} \Delta V_{\|, i}+S_{v_{\|}} \\
\vec{e}_{\varphi} \cdot \nabla \times\left(\rho \frac{\partial \vec{V}_{E}}{\partial t}=-\rho(\vec{V} \cdot \nabla)\left(\vec{V}_{\|, i}+\vec{V}_{E}\right)\right. \\
\left.+\vec{J} \times \vec{B}-\nabla P-\bar{\nabla} \cdot \bar{\Pi}_{i, n e o}+\mu_{\perp} \Delta V\right) \\
\rho \frac{\partial T}{\partial t}=-\rho \vec{V} \cdot \nabla T-(\gamma-1) P \nabla \cdot \vec{V}+\frac{1}{2} V^{2} S_{\rho} \\
+\nabla \cdot\left(\kappa_{\|} \nabla_{\|} T+\kappa_{\perp} \nabla_{\perp} T\right)+(1-\gamma) S_{T} \\
\frac{1}{R^{2}} \frac{\partial \psi}{\partial t}=-\vec{B} \cdot \nabla_{\|} u+\frac{\tau_{I C}}{\rho} \vec{B} \cdot \nabla_{\|} P+\frac{\eta J}{R^{2}}
\end{gathered}
$$

where the parallel gradient is defined as

$$
\begin{aligned}
\nabla_{\|} \alpha & =\left(\vec{B} / B^{2}\right) \vec{B} \cdot \nabla \alpha \\
& =\frac{\vec{b}}{B}\left(\frac{F_{0}}{R^{2}} \partial_{\varphi} \alpha+\nabla \varphi \cdot \nabla \alpha \times \nabla \psi\right)
\end{aligned}
$$

The normalization of the variables in JOREK units (noted with superscript $\sim$ ) is the following: time is defined as $t=\tilde{t} \sqrt{\rho_{0} \mu_{0}}$, mass density is $\rho=\tilde{\rho} \rho_{0}$, temperature is $T(e V)=m_{i} /\left(\rho_{0} \mu_{0} e\right) \tilde{T}$, the total pressure is $\tilde{P}=\mu_{0} P=\tilde{\rho} \tilde{T}$ and the toroidal current is $\tilde{J}=\mu_{0} J$. Note that the toroidal current density is defined as $j=\Delta_{\perp} \psi=-R^{2} \vec{J} \cdot \nabla \varphi$ and hence in JOREK system 
a co-current injection corresponds to a negative source of parallel velocity. The electrostatic potential $\tilde{u}$ is related to the electric field as $\vec{E}=-F_{0} \nabla \tilde{u} / \sqrt{\rho_{0} \mu_{0}}$. For each species $s$, the parallel and perpendicular components of the velocity are $\vec{V}_{\|, s}=\tilde{V}_{\|, s} \vec{B} / \sqrt{\rho_{0} \mu_{0}}\left(\tilde{V}_{\|, s}\right.$ has no dimension) and $V_{\perp, s}=\tilde{V}_{\perp, s} / \sqrt{\rho_{0} \mu_{0}}\left(\tilde{V}_{\perp, s}\right.$ is expressed in Tesla). Once normalized, the decomposition of the fluid (ion) velocity (1) therefore becomes:

$$
\vec{V}=V_{\|, i} \vec{B}-R^{2} \nabla u \times \nabla \varphi-\frac{R^{2} \tau_{I C}}{\rho} \nabla P \times \nabla \varphi
$$

where the inverse of the normalized ion cyclotron frequency is defined as $\tau_{I C}=m_{i} /\left(2 F_{0} e \sqrt{\rho_{0} \mu_{0}}\right)$. The electron and ion temperatures $T_{e}$ and $T_{i}$ are assumed to be equal in the model used here.

The projection of equation (11) along the poloidal direction $\vec{e}_{\theta}(3)$ gives the expression of the poloidal velocity:

$$
V_{\theta}=-\frac{1}{B_{\theta}}\left(\nabla_{\perp} \psi \cdot \nabla_{\perp} u+\frac{\tau_{I C}}{\rho} \nabla_{\perp} \psi \cdot \nabla_{\perp} P-V_{\|, i} B_{\theta}^{2}\right)
$$

The combination of equations (12) and (3) with the expression of the normalized neoclassical velocity $V_{\theta, \text { neo }}=$ $-\frac{k_{i} \tau_{I C}}{B_{\theta}}\left(\nabla_{\perp} \psi \cdot \nabla_{\perp} T\right)$ leads to the developed form of the neoclassical tensor (2):

$$
\begin{aligned}
& \nabla \cdot \bar{\Pi}_{i, \text { neo }}=\mu_{i, n e o} \rho \frac{B^{2}}{B_{\theta}^{4}}\left(-V_{\|, i} B_{\theta}^{2}+\nabla_{\perp} \psi \cdot \nabla_{\perp} u\right. \\
& \left.+\frac{\tau_{I C}}{\rho}\left(\nabla_{\perp} \psi \cdot \nabla_{\perp} P+k_{i} \rho \nabla_{\perp} \psi \cdot \nabla_{\perp} T\right)\right)(\nabla \psi \times \nabla \varphi)
\end{aligned}
$$

Realistic radial profiles of the neoclassical parameters $\mu_{i, n e o}$ and $k_{i}$ (calculated from [21]) which depend both on the aspect ratio and on the collisionnality, are used in the simulations for the JET case III. For the sake of simplicity, these coefficients are taken constant in the ITER case $\mathrm{IV}$, where typical values $\tilde{\mu}_{i, \text { neo }}=\mu_{i, \text { neo }} \sqrt{\rho_{0} \mu_{0}}=10^{-5}$ and $k_{i}=-1$ [12] are used. In the MAST case, the neoclassical friction is not included in the model.

$D_{\perp}$ is the perpendicular particle diffusion, $\mu_{\|}$and $\mu_{\perp}$ are the parallel and perpendicular anomalous viscosity coefficients, $\gamma=5 / 3$ is the adiabatic index, $\kappa_{\|}$ and $\kappa_{\perp}$ are the parallel and perpendicular heat diffusivity and $\eta$ is the resistivity. These parameters are normalized as $\tilde{D}_{\perp}=D_{\perp} \sqrt{\rho_{0} \mu_{0}}, \tilde{\kappa}_{(\|, \perp)}=\kappa_{(\|, \perp)} \sqrt{\rho_{0} \mu_{0}}$, $\tilde{\mu}_{(\|, \perp)}=\mu_{(\|, \perp)} \sqrt{\mu_{0} / \rho_{0}}$ and $\tilde{\eta}=\eta \sqrt{\rho_{0} / \mu_{0}}$. Both viscosity and resistivity follow a Spitzer-like $\left(T / T_{0}\right)^{-3 / 2}$ dependence and the parallel heat diffusivity varies as $\left(T / T_{0}\right)^{5 / 2}$. In the core plasma, for a particle density $n_{0}=6 \times 10^{19} \mathrm{~m}^{-3}$ (JET case), the typical values used in simulations are $D_{\perp}=\kappa_{\perp}=2 \mathrm{~m}^{2} / \mathrm{s}, \kappa_{\|} / \kappa_{\perp}=2 \times 10^{8}$, $\mu_{\perp}=4 \times 10^{-7} \mathrm{~kg} /(\mathrm{m} . \mathrm{s}), \mu_{\|} / \mu_{\perp}=10$ and $\eta \sim 10^{-7} \Omega . \mathrm{m}$.

$S_{\rho}, S_{V_{\|}}$and $S_{T}$ are sources of particles, parallel momentum and heat respectively. The source of parallel rotation $\tilde{S}_{v_{\|}}=-\tilde{\mu}_{||} \Delta \tilde{V}_{\|, t=0}$ forces the parallel velocity close to the experimental rotation profile and compensates the losses due to the parallel viscosity. Reproducing realistic sources of heat and particle is not a simple issue, so the particle source is set constant in the plasma and the radial profile of the heat source follows the temperature profile.

At the boundary of the computational domain, Dirichlet conditions are used for all the variables, except for the parallel velocity, temperature and density on the divertor targets. There, Bohm conditions are set (the parallel velocity is equal to the sound speed $C_{s}$ ) and the temperature and density outflow is left free. Consequently the parallel and perpendicular flows self-consistently evolve towards an equilibrium both in the bulk plasma and the SOL, since they are constrained by Bohm conditions, neoclassical and diamagnetic effects and the source of parallel rotation. This results in the self-consistent evolution of the radial electric field, expressed by the radial force balance (in JOREK units):

$$
E_{r}=\frac{\tau_{I C}}{\rho} \frac{\nabla_{\perp} \psi \cdot \nabla_{\perp} P}{|\nabla \psi|}+\frac{1}{F_{0}}\left(V_{\theta} B_{\varphi}-B_{\theta} V_{\varphi}\right)
$$

The equilibrium flows are established in a time scale of $\sim 10^{3}$ Alfven times $t_{A}$. Once they are obtained, perturbation modes $(n \neq 0)$ including the perturbation due to RMPs are added to the simulation. In the simulations described below, only the fundamental mode $n=0$ and the toroidal harmonic corresponding to the symmetry of the EFCC $(n=2$ in JET) or the RMP coils $(n=3$ in ITER and MAST) are included. The interaction of the "RMP-mode" with unstable modes (ELMs) will be studied in a future work. The application of the RMPs is modeled by a change in boundary conditions for the magnetic flux perturbation. The vacuum RMP spectrum is previously calculated with the ERGOS code [22] and applied as boundary conditions of the computational domain for the magnetic flux perturbation. RMPs are progressively switched on in time: the amplitude of the perturbation is gradually increased in the typical timescale $t \sim 1000 t_{A}$. That way, the magnetic perturbation gradually penetrates inside the plasma, which self-consistently adapts in the process. Also this method avoids possible problems with hysteresis effects that could occur if the simulation was started with a fully penetrated magnetic perturbation [23]: the bifurcation from a fully penetrated state to a partially penetrated state might lead to a different plasma response.

\section{JET-LIKE STUDY}

\section{A. Input parameters}

In this section, JOREK is initialized with JET-like plasma parameters: $R=3 m, a=1 m, B_{t}=2.9 T, q_{95} \sim 3$ in toroidal geometry including the $\mathrm{X}$-point. Typical $\mathrm{H}-$ mode density and temperature profiles are chosen: the 
central electron density is $n_{e, 0}=6 \times 10^{19} \mathrm{~m}^{-3}$, the central temperature is $T_{e, 0}=5 \mathrm{keV}$ and the central toroidal rotation is $\Omega \sim 9 k H z$. Input profiles are given in Fig.1. Realistic profiles of the neoclassical coefficients are used in this section, given in Fig.2. The RMP spectrum generated by the external Error Field Correction Coils (EFCC) is considered in the simulations, with the following configuration: a toroidal symmetry $n=2$ is taken and a current $I_{\text {coil }}=40 k A t$ is injected in the EFCC. The vacuum magnetic flux perturbation calculated with ERGOS is given in Fig.3.

\section{B. Equilibrium plasma flows}

The modeling of the plasma flows in a consistent way is primordial since they impact the penetration or the screening of the RMPs. In this subsection, equilibrium plasma flows are first studied without RMPs, in order to highlight the patterns generated by the different effects both in the scrape-off layer and the pedestal, in the poloidal and toroidal directions.

In the SOL, the poloidal and toroidal flows are mainly set by the sheath boundary conditions: the parallel Mach $=1$ condition $\left(V_{\|}= \pm C_{s}\right.$, where $C_{s}$ is the ion sound speed) set on the divertor targets results in the evolution of the parallel velocity in the SOL until an equilibrium parallel flow is reached, characterized by an opposite direction in the low and high field sides and a bounce point located at the top of the SOL (Fig.4). The divergence-free condition of the velocity thus constrains also the poloidal flow pattern (Fig.5) in the SOL.

As for the central plasma, the parallel velocity (Fig.4) is constrained by the source of parallel velocity which mimics the experimental profiles. In the poloidal direction, the flows (Fig.5) are close to their neoclassical prediction (Fig.6), especially in the strong temperature gradient pedestal region where neoclassical damping is strong.

The radial electric field $E_{r}$ (Fig.7) resulting from the equilibrium force balance (14) adapts accordingly. In particular, in the pedestal, a deep electric field well is generated by the diamagnetic terms (Fig.7), reaching up to $-10^{5} \mathrm{~V} / \mathrm{m}$, which is in the ballpark of the values measured in the experiments [24]. The $E_{r}$ well obtained in our simulations is five times deeper than in the pulse considered in [24], but this is consistent with the fact that the pedestal is much higher in our case and thus diamagnetic effects are larger. Indeed, the values of the density $\left(5 \times 10^{19} \mathrm{~m}^{-3}\right)$ and temperature $(2 \mathrm{keV})$ at the top of the pedestal are 2-3 times larger in the simulation than in the experimental pulse [24]. We can also note in Fig.7 that the toroidal source of rotation $\left(f_{\text {tor }}=9 \mathrm{kHz}\right) \mathrm{com}-$ bined with the neoclassical effects make the radial electric field positive in the core. Indeed, in the core plasma, the pressure gradient is close to zero and the poloidal velocity is close to the neoclassical value (proportional to the low temperature gradient), so the radial electric field approx- imates $E_{r}=-B_{\theta} V_{\varphi}$ in order to satisfy the radial force balance (14). The radial electric field is thus positive in the core and negative in the pedestal, so the perpendicular electron velocity $V_{\perp, e}=V_{E \times B}+V_{e}^{*}$ (depending on $E_{r}$ ) is zero on a particular surface at the top of the pedestal. If this surface is a rational surface $(q=m / n$, $n$ being the mode driven by the RMPs), the magnetic perturbation is likely to fully penetrate $[11,12,25]$. The radial profiles of the parallel, poloidal, neoclassical and perpendicular velocities and the radial electric field are given in Fig.8 (Low Field Side) and Fig.9 (High Field Side) at the midplane.

\section{RMP screening by large diamagnetic rotation and low resistivity}

Once equilibrium flows are established, $\operatorname{RMPs}(n=2$, generated by EFCC, $\left.I_{\text {coil }}=40 k A t\right)$ are progressively increased at the boundary in $\sim 1000 t_{A}$. Without RMPs, the mode $n=2$ is marginally stable (Fig.10). In the presence of RMPs, the flux of magnetic perturbation (Fig.11) penetrates inside the plasma: the energy of the $n=2$ toroidal mode grows, until a quasi-steady state is reached (Fig.10). On the resonant surfaces $(q=m / n, n=2, m \geq$ 3 ), magnetic islands grow up to the saturation, due to reconnection forced by magnetic perturbations. Parallel current perturbations (Fig.12) are induced on these resonant surfaces in response to the magnetic perturbations and allow for the screening of the RMPs: if the response current is in phase with the radial magnetic perturbation on a given resonant surface, the corresponding RMP harmonic is screened. If the magnetic and current perturbations are in antiphase, the magnetic perturbation penetrates on the corresponding resonant surface as in vacuum and can even be amplified [12].

These induced currents depend both on the plasma flows and on the resistivity, so the different terms influencing the flows (diamagnetic velocity, neoclassical friction and source of parallel rotation) and affecting the plasma response to RMPs are progressively added in the simulation. The central resistivity is scanned between $\eta_{0}=10^{-7}$ and $\eta_{0}=5 \times 10^{-8}$ (in JOREK units). Note that for numerical reasons these values are taken $1-2$ orders of magnitude larger than the realistic (Spitzer) value in $\operatorname{JET}\left(\sim 10^{-9}\right)$.

The effects of the diamagnetic flow and the resistivity are first studied ; the source of parallel momentum and then the neoclassical friction are added in the model over a second phase. In all the studied cases, the central harmonic of the perturbation located on the resonant surfaces $q=3 / 2$ is screened. The Poincaré plot on the magnetic field (Fig.13, here for low poloidal rotation $\tau_{I C}=10^{-3}$ and high resistivity $\eta_{0}=10^{-7}$ ) shows that small islands are generated on the resonant surface $q=4 / 2$ : this means that the screening of the ( $m=4, n=2$ ) harmonic is only partial. However the magnetic perturbation mostly penetrates at the very edge 
of the plasma where the resistivity is higher (Fig.13). In these simulations, the magnetic shear is strong for $q \geq 2.5$ (Fig.1) so the proximity of the surfaces $q=5 / 2$ and $q=6 / 2$ explains the formation of a stochastic layer at the edge, for $\psi \geq 0.97$ (Fig.13). Yet, since the modes $m \geq 6$ are located too close to the separatrix, only the mode $m=5$ seems to develop significantly, as suggests the structure of the density and temperature perturbations presented in Fig.15 and Fig.16. It is also interesting to note that the coupling of the modes generates secondary islands on the rational surface $q=9 / 4$ and even tertiary islands on $q=13 / 6$ (Fig.13). The observation of these secondary and tertiary islands can be explained by the flat q-profile in this area (Fig.1), since the island size is inversely proportional to the magnetic shear $d q / d \psi$.

From the resistivity scan and the progressive addition of the different flows, we find that the diamagnetic rotation and the resistivity are the two main parameters affecting the penetration of the RMPs, and three different regimes are identified depending on these two parameters (Fig.17).

At low diamagnetic rotation $\left(\tau_{I C}=10^{-3}\right.$, which is half the realistic value) and high resistivity $\left(\eta_{0}=10^{-7}\right)$, the generated islands rotate in the ion diamagnetic direction at the ion poloidal rotation frequency $f^{*} \sim m \frac{V_{\theta}}{2 \pi r_{r e s}}$, where $m$ is the poloidal mode number corresponding to the resonant surface. The main poloidal mode generated being the mode $m=5$ (Fig.15 and Fig.16), the oscillation of the magnetic energy of the mode $n=2$ (Fig.17) mainly corresponds to the rotation of the magnetic islands on the surface $q=5 / 2$ at the frequency $f^{*} \sim 6 k H z$. The amplitude of these islands oscillates at the same frequency $f^{*}$ : indeed, while they are rotating with the plasma, they successively face maxima and minima of external static magnetic perturbations, which explains the fluctuation of the island size at the frequency $f^{*}$. This mode is probably related to the Rutherford regime described in the Fitzpatrick theory [16] and such an oscillating regime is also found in simulations with toroidal rotation included [26]. Small fluctuations of the SOL density and temperature are observed in this regime to rotate at the same frequency as the magnetic flux fluctuations (Fig.18, solid line). Note that the volume integrated density and temperature in the SOL increase when switching on the RMPs, suggesting an enhancement of the density and heat transport due to the formation of an ergodic region at the edge (Fig.13). However no significant changes in plasma density and temperature profiles were observed in modeling ; the density pump-out has not been observed so far: less than $1 \%$ of the pedestal density is lost in the simulations, as compared to up to $20 \%$ in the experiments $[3,6]$. The regime of oscillating islands is possibly related to the ELM suppression regimes at high collisionality (characterized by a smaller pedestal, a smaller diamagnetic rotation and a high resistivity [4, 27]), but more modeling of realistic experimental cases is needed to confirm this statement.

At larger diamagnetic rotation $\left(\tau_{I C}=2 \times 10^{-3}\right.$, cor- responding to the realistic value) and high resistivity $\left(\eta_{0}=10^{-7}\right)$, the driven mode is on the contrary static and "locked" to the external RMPs. The Poincaré plot (Fig.14) shows that the islands generated on the rational surface $q=4 / 2$ are smaller at larger $\tau_{I C}$, which means that the RMPs are more screened by the diamagnetic flow. As for the secondary and tertiary islands, they are totally invisible in this case. Consistently, the magnetic energy of the mode $n=2$ is decreased as compared to the oscillating case (Fig.17). Nonetheless, an ergodic layer is formed at the very edge (for $\psi \geq 0.98$ ), but its width is smaller and the deformation of the boundary is reduced. The heat transport, slightly enhanced by RMP application (as suggested by the increase in SOL temperature in Fig.18, dash line), is weak compared to the previous case, due to the smaller ergodization of the edge.

An intermediate regime is also found at lower resistivity $\left(\eta_{0}=5 \times 10^{-8}\right)$ with $\tau_{I C}=10^{-3}$ : the magnetic islands are quasi-static and slightly oscillate at the ion poloidal rotation frequency. The screening level is approximately the same as in the static case.

RMP screening is quantified in these three cases in Fig.19, where the Fourier harmonics $(n=2, m=3-6)$ of the magnetic flux perturbation are plotted. In the three cases, the central islands $(m=3-4)$ are screened on the corresponding resonant surfaces. Yet the Fourier harmonics $\left|\psi_{m n}\right|(m=3-4)$ do not totally vanish on the resonant surfaces (which is consequent with the formation of small islands on $q=4 / 2$ ), contrary to the previous modeling in cylindrical geometry [12] where the central harmonics were totally screened. The discrepancy between cylindrical and toroidal modeling is not really understood, but will be discussed in section IV. Regarding the edge harmonics $(m=5-6)$, the amplitude of the Fourier harmonics $\left|\psi_{m n}\right|$ is 2-3 times lower in the cases at low resistivity or high diamagnetic rotation than it is in the oscillating case, which means that the corresponding island size, proportional to $\left|\psi_{m n}\right|^{1 / 2}$, is reduced.

From now, the source of parallel momentum is added in the model. If the screening effect provided by high diamagnetic rotation and/or low resistivity is clear in the JET case, the effect of the parallel rotation is more complex (Fig.20). As the parallel rotation does not directly affects the screening of RMPs, an increased parallel rotation does not necessarily imply an increased screening of RMPs. Indeed, at low resistivity $\left(\eta_{0}=5 \times 10^{-8}\right)$, in the slightly oscillating regime characterized by low diamagnetic rotation $\left(\tau_{I C}=10^{-3}\right)$, the parallel rotation increases both the average island size and the fluctuation of the island width: this can explain the larger fluctuation of the magnetic energy of the mode $n=2$ in Fig. 20 . The oscillation frequency of the islands is also modified by the parallel rotation, probably because of the induced change in the radial electric field and the poloidal rotation. On the contrary, at higher diamagnetic rotation $\left(\tau_{I C}=2 \times 10^{-3}\right)$, the energy of the mode $n=2$ is lower when the parallel velocity source is added, showing a slightly stronger screening by parallel velocity. 
Last, in our simulations with the realistic diamagnetic rotation $\left(\tau_{I C}=2 \times 10^{-3}\right)$ and the source of parallel rotation (at $\eta_{0}=10^{-7}$ ), the addition of the neoclassical effects in the model modifies the plasma response. For a same simulation without neoclassical friction, the magnetic islands generated on $q=5 / 2$ are static; however with neoclassical effects the generated islands are first static and then progressively rotate in the ion flow direction, until they reach the ion perpendicular rotation frequency $f^{*}$ (Fig.21). The island width also oscillates at the frequency $f^{*}$. We are thus in the same regime of plasma response as for the case at low diamagnetic rotation. The change in the regime of plasma response is not due to a modified perpendicular rotation (in both cases without and with neoclassical effects, the perpendicular velocity is the same at the vicinity of the resonant surface $q=5 / 2$ ). The reason why this regime is obtained at large diamagnetic rotation with neoclassical friction is not really understood, but it may be explained by a change in viscosity resulting from the balance between the neoclassical friction force and the viscous force in the momentum equation. More modeling would be necessary to give a global interpretation of the phenomena at stake. The Poincaré plot of the magnetic topology of this case is given in Fig.22. Compared to Fig.14 (case without source of parallel rotation or neoclassical friction), the island width and the size of the ergodic layer is similar. Actually, in all cases, the position where perpendicular velocity cancels (red dashed line on Fig.22) is located between $q=4 / 2$ and $q=5 / 2$ and does not match a resonant surface. It explains why the perturbations significantly penetrate only at the very edge $(\psi \geq 0.97)$ where resistivity is higher and where the perpendicular rotation is dominated by the diamagnetic effects.

\section{RMPS IN ITER}

\section{A. Input parameters and equilibrium plasma flows}

In this section, the equilibrium and plasma parameters of the ITER standard H-mode scenario $15 M A / 5.3 T$ are used. The machine dimensions are $R=6.2 \mathrm{~m}, a=2 \mathrm{~m}$ and the safety factor is $q_{95}=3.5$. The density, temperature and toroidal rotation profiles are given in Fig.23. Central density is $n_{e, 0}=8 \times 10^{19} \mathrm{~m}^{-3}$, central temperature is $T_{0}=27.8 \mathrm{keV}$ and the initial toroidal rotation profile is similar to the temperature profile with a central arbitrary rotation frequency $\sim 0.9 k \mathrm{~Hz}$, which is in the range of the expected value in ITER. The flux-aligned mesh used for modeling is given in Fig.24. The resonant magnetic flux perturbations generated by In-Vessel Coils, in the configuration $n=3$ and a current $I_{\text {coil }}=54 \mathrm{kAt}$ (Fig.25, [9]) are calculated from the vacuum code ERGOS [22] and are applied as boundary conditions in $10^{3} t_{A}$ in the same way as in the previous section.

Equilibrium plasma flows are computed for a central resistivity $\eta_{0}=10^{-8}$, which is, for numerical reasons, 100 times higher than the normalized Spitzer value calculated for ITER $\left(\eta_{0}=10^{-10}\right)$. As the diamagnetic parameter is inversely proportional to $F_{0}=R_{0} B_{\varphi 0}$, the value for ITER $\tau_{I C}=5 \times 10^{-4}$ is 4 times smaller than in the JET simulation. Neoclassical coefficients are taken constant in this part for simplicity: $\mu_{n e o}=10^{-5}$ and $k_{i}=-1$ [12]. The profiles of the parallel, poloidal and neoclassical velocities along the midplane are given in Fig.26. The equilibrium radial electric field $E_{r}=-\nabla_{\perp} u \cdot \nabla \psi /|\nabla \psi|$, with the characteristic well in the pedestal, is plotted in Fig.27.

\section{B. RMP screening in ITER}

When the RMPs are not activated, the mode $n=3$ remains stable: as shown in Fig.28, the magnetic energy of the mode $n=3$ is negligible. When RMPs are switched on (Fig.25) at the boundary of the computational domain, the energy of the $n=3$ toroidal harmonics grows until it saturates, corresponding to the saturation of the size of the magnetic islands generated by the RMPs on the resonant surfaces $q=m / 3, m \geq 4$. These magnetic islands are static: they are "locked" to the external RMPs and their size is constant. The magnetic flux perturbation $n=3$ is given in Fig.29. The plasma response is manifested by a perturbation of the parallel current induced on the resonant surfaces (Fig.30). The perturbation of density (Fig.31) and temperature (Fig.32) mainly develops at the very edge $(q=m / 3, m \geq 10)$ where the resistivity is higher ; on the more internal resonant surfaces $(q=4 / 3 \rightarrow 9 / 3)$, the perturbation vanishes, which means that the central harmonics are screened. The temperature and density perturbations and the stochastization of the edge (for $\psi \geq 0.96$, as shown on the Poincaré plot Fig.36) slightly increase the edge heat and particle transport, resulting in a small degradation of the pedestal (Fig.33).

The profiles of the Fourier coefficients of the magnetic perturbation $\psi_{m, n}$ calculated with JOREK in presence of the plasma response are compared to the coefficients calculated in the vacuum with the code ERGOS. Compared to the vacuum case [12], the central harmonics $m=4-9$ (Fig.34, Fig.35) are screened by the rotating plasma on the corresponding resonant surfaces $q=m / n$. Yet it is important to note that the screening of the resonant part of the magnetic perturbations is only partial and that the screening factor $\left|\psi_{m, n}\right| /\left|\psi_{m, \text { nvacuum }}\right|$ on the resonance reaches $\sim 10 \%$ for $m=4-9$. This partial penetration is consequent with the fact that small magnetic islands are formed on the resonant surfaces (Fig.36). The screening factor is much larger here than in the cylindrical modeling where the resonant component was reduced under 1\% [12]. Comparatively, other simulations made with toroidal codes $[13,14]$ also found a similar screening factor $(\sim 10-20 \%)$, which is $1-2$ orders of magnitude larger than the results in cylindrical approximation $[11,12,28]$ and in the analytical calculations from layer 
theory [29]. The discrepancy between modeling in realistic geometry and simpler models is not yet understood. Several parameters such as toroidal effects, compressibility or differences in resistivity and viscosity might explain this discrepancy. Another phenomenon pointed out in toroidal simulations (Fig.34, Fig.35 and [14]) is the amplification of the non-resonant harmonics due to the so-called resonant field amplification (RFA). Indeed, the amplitude of the magnetic perturbations in the core is larger with plasma response than in the vacuum modeling, due to the resonant response of a marginally stable kink mode [30]. At the edge, the amplitude of the resonant harmonics $m=10-11$ (Fig.35) have the same order of magnitude in both vacuum and plasma cases. The magnetic perturbation thus significantly penetrates only at the very edge where the resistivity is the highest, which is similar to the cylindrical non-linear MHD modeling results [12].

\section{Stochasticity at the edge}

The RMP penetration at the edge generates the formation of islands on the resonant surfaces $q=m / 3, m \geq 10$. As the edge resonant surfaces are close to each other due to strong magnetic shear, the islands overlap and therefore form an ergodic layer at the edge for $\psi \geq 0.96$, as suggests the Poincaré plot of the magnetic topology in $(\psi, \theta)$ coordinates Fig.36.

The formation of the ergodic layer is correlated with a splitting of the separatrix [31]. The separatrix under the RMP transforms into a homoclinic tangle defining the plasma edge [32], manifesting itself as lobe structures $(\sim 20 \mathrm{~cm})$ near the X-point (Fig.37) and by smaller finger-like structures $(\sim 6 \mathrm{~cm})$ at the top of the plasma (Fig.38). The superposition of the magnetic topology with the plot of the homoclinic tangles (stable and unstable) defining the deformation of the separatrix are given in Fig.39. These lobes near the X-point are observed in the experiments, either directly ([33], [34]) or as the footprints of high heat and/or particle fluxes where the lobes intersect the divertor [35-38]. Models of plasma response based on a simplified assumption of screening currents on resonant surfaces [39] or on a linear MHD model [40] predict significant shortening of the lobes when the RMP is screened by the plasma response. Shortening is observed in the magnetic field topology [38-40] and through the reduction of fluxes [41, 42] and generally increases with the increase of the width of the plasma region where the RMP is screened [39]. The shortening of lobes currently represent the most convenient way to quantify experimentally the screening of RMPs. In the same way, in our simulations, the size of the lobes qualifies the screening of the RMPs by plasma flows. In Fig.37, a simulation with the same input RMP amplitude is run in two cases: with SOL flows only (induced by sheath conditions) and with all flows included (neoclassical and diamagnetic flows, source of toroidal rotation). The ad- dition of the flows in the simulation shortens the length of the lobe structures near the X-point by $\sim 1 / 3$, which corroborates the hypothesis (so far deduced from simpler models) that the RMP screening by plasma flows is correlated to lobe/footprint shortening.

A 3D-deformation of the density, temperature and pressure profiles is observed. The deformation of the pressure profile close to the separatrix is found to be due to the mode $(n=3, m \sim 12)$, Fig.40. A small degradation of the density and temperature profiles is also observed near the X-point when RMPs are applied. The comparison of the density profile with (Fig.41) and without RMPs (Fig.42) shows the enhancement of the particle transport towards the divertor targets by the RMPs. A similar enhancement of the heat transport is observed. This increase in heat and particle fluxes subsequently generates a small splitting of the strike points on the divertor targets, mostly on the outer target (Fig.43).

\section{FIRST SIMULATIONS IN MAST DND CONFIGURATION}

\section{A. Input parameters}

RMP penetration in MAST is studied in Double Null Divertor configuration with plasma parameters corresponding to the shot \#24763. The central density is $n_{e, 0}=4.25 \times 10^{19} \mathrm{~m}^{-3}$, the central electron temperature is $T_{e, 0}=1.04 \mathrm{keV}$ and the central resistivity is taken $\eta_{0}=10^{-7}$ (this is two orders of magnitude larger than the normalized Spitzer value). The disposition of the RMP coils chosen is the "90L" configuration, since it corresponds to a maximal amplitude of the radial magnetic field (generated by the RMPs) located on the resonant surfaces, as shown in Fig.44. The $n=3$ Fourier component of the vacuum RMP field imposed at the boundary of the computational domain is given in Fig.45. For numerical reasons, in the simulation the current circulating in the coils is taken to be $1 / 10$ of the experimental value.

Two different cases are studied and compared: first, the simulation is run with only the SOL flows generated by sheath conditions. Second, the diamagnetic effects are added, with a diamagnetic parameter $\tau_{I C}=10^{-2}$ (note that the large rotation in MAST implies a realistic diamagnetic parameter $\tau_{I C}=5 \times 10^{-2}$, ie 100 times the one of the ITER case, but a lower value is taken here for numerical reasons). In this section, neither the source of parallel rotation nor the neoclassical poloidal friction are included in the model so as to focus on the effects of the diamagnetic rotation on RMP penetration.

\section{B. RMP penetration}

In both cases (with or without diamagnetism), a static $n=3$ mode grows due to the RMPs (Fig.46). The magnetic energy of the mode is a few percent higher in the 
case including the diamagnetic flow, which means that the diamagnetic effects slightly amplify the magnetic perturbations in this MAST configuration.

The penetration of the magnetic flux perturbation is given in Fig.47 and the induced current perturbations on the resonant surfaces is shown in Fig.48 (case without diamagnetic flow). The electron density (Fig.49) and temperature perturbations (Fig.50, $n=3$ ) represent up to $10 \%$ of the central average value and are mainly located at the edge on the LFS, at the proximity of the RMP coils.

The radial profiles of the Fourier harmonics of the magnetic flux perturbation $\left|\psi_{m, n}\right|(n=3, m=4-12)$, are presented Fig.51 and Fig.52 for both cases with and without diamagnetic flow. In both cases, the central resonant harmonics $m=4-9$ are screened near the corresponding surfaces $q=m / n$. The screening of the resonant harmonics is only partial (reduced to $\sim 10 \%$ of the vacuum perturbation) and magnetic islands are thus generated on the resonant surfaces in both cases (without Fig. 53 and with diamagnetic effects Fig.54). These islands are larger in the core plasma in the simulation without diamagnetic rotation: the amplitude of the low poloidal numbers is reduced by the rotation. Also the amplification of the nonresonant magnetic perturbation due to RFA is observed in the core. At the edge, the harmonics $m=10-12$ penetrate in both cases, but the amplitude is larger in the diamagnetic case (Fig.52): the diamagnetic flow seems to amplify the perturbation at the edge. Indeed the resonant components of the Fourier harmonics $m=9-12$ are slightly larger with diamagnetic effects on the corresponding resonant surfaces $q=m / n$ (Fig.53-Fig.54).

\section{Ergodization and 3D-effects}

The penetration of the external RMP harmonics creates an ergodic layer at the edge for $\psi \geq 0.95$ (Fig.53Fig.54). Related to the ergodization are lobe structures formed near the X-point, as shown in Fig.55 (case with diamagnetic flows included). It is interesting to notice that the presence of the two separatrices in DND configuration constrains the trajectories of the magnetic field lines so the lobes are only formed in the LFS. This phenomenon is also observed in MAST DND experiments with the fast camera. The size of the lobes in the LFS are comparable in both studied cases (with and without diamagnetic effects).

The 3D-deformation following a $n=3$ toroidal mode number is observed in both simulations in the LFS. The separatrix is displaced towards the interior by a few centimeters at the midplane: $\sim 2-3 \mathrm{~cm}$ without diamagnetism and $\sim 1.5 \mathrm{~cm}$ in the diamagnetic case (Fig.56) for a toroidal angle $\varphi=0^{\circ}$. The inward displacement of the electron density and temperature profiles at the midplane in the LFS (Fig.57) is maximal for a toroidal angle $\varphi=0^{\circ}$ (position where the RMP coils deliver the largest negative flux perturbation) and minimal for $\varphi=180^{\circ}$ (angle where the maximal positive flux perturbation is applied). Compared to the density and temperature profiles without RMPs, the gradients are lower in the pedestal when the RMPs are applied, showing a small degradation of the confinement by the RMPs: this can be explained by the enhancement of the heat and particle transport due to the ergodicity at the edge. The 3D-corrugation of the electron density and temperature profiles are observed: the deformation of the temperature near the separatrix is given in Fig. 58 .

Last, it is observed in the experiments that the radial electric field is made more positive by RMP application ([43]). This phenomenon was found in simulations in the cylindrical case ([12]) and is also found in our toroidal simulations with JOREK, as plotted in Fig.59 in the LFS. The braking of the perpendicular electron velocity $V_{\perp, e}$ induced by RMPs is also evidenced in JOREK simulations Fig.60, following the same trend as in the experiments ([44], [34]). If the plasma brakes until $V_{\perp, e}$ becomes zero on the resonant surfaces, the magnetic perturbations are not screened on the resonant surfaces, as it was found in [11], [12] and [25] in cylindrical geometry and here demonstrated in Appendix for toroidal geometry.

It is important to note that this study for the MAST case is a preliminary study, and can only give trends of the effect of RMPs on the plasma in this particular case of a spherical tokamak in DND configuration. Clearly, the size of the displacements calculated here cannot be quantitatively compared to the experiments, since numerical limits imposed us to "minimize" two antagonist effects: on the one hand, the realistic RMP amplitude (10 times the one used in our simulations) should generate much larger penetration, on the other hand the realistic diamagnetic rotation ( 5 times the one used here) is likely to have much larger screening effects on the RMPs. Anyway these results can be compared qualitatively to the experimental trends.

\section{CONCLUSION AND DISCUSSION}

The penetration of the RMPs in the plasma was studied with the reduced MHD code JOREK, taking into account the plasma flows generated by the diamagnetic and neoclassical effects and a source of toroidal rotation. The screening of the RMPs on the resonant surfaces is observed in the center of the plasma in all the studied cases (JET, ITER, MAST) but the screening is only partial. The factor $\left|\psi_{m, n}\right| /\left|\psi_{m, n, v a c u u m}\right|$ on the resonance is 1-2 orders of magnitude larger than in the previous cylindrical modeling. The perturbation however significantly penetrates at the edge: magnetic islands grow on the resonant surfaces and eventually form an ergodic layer at the edge (for $\psi \geq 0.95$ in the three studied cases).

In JET geometry, a low plasma resistivity and a large diamagnetic rotation are found to increase the screening of the RMPs. Three regimes of RMP penetration 
are found depending on these two parameters: at low diamagnetic rotation and high resistivity, the generated islands co-rotate with the plasma ion flow and their size fluctuate at the same frequency. At larger diamagnetic rotation, the islands are static and "locked" to the external static RMPs; a stronger screening of the perturbations is observed. An intermediate regime is observed at lower resistivity, in which the generated island are quasistatic and slightly oscillate. The possible link between these regimes and the difference in ELM mitigation at high and low collisionality [3, 27] is under investigation. As for the toroidal rotation, depending on the plasma parameters (in particular on the resistivity), it can either increase or amplify the screening of the RMPs. Also, contrary to the JET case, the magnetic perturbation seems to be slightly amplified by the diamagnetic rotation at the edge in MAST simulations.

In MAST and ITER cases, static magnetic islands are formed on the resonant surfaces due to $n=3$ magnetic perturbations. The presence of lobe structures related to the ergodization at the edge are evidenced in both cases $(\sim 20 \mathrm{~cm}$ in ITER, $\sim 7 \mathrm{~cm}$ in MAST). In ITER, the comparison of simulations run with and without flows demonstrates that the plasma rotation reduces the length of the lobes by $1 / 3$. The heat and particle transport is enhanced by the ergodicity near the $\mathrm{X}$-point, resulting in the local degradation of the electron density and temperature profiles and the splitting of the strike points on the divertor targets, mostly in the LFS. In MAST, the separatrix follows a $(n=3, m \sim 11) 3 \mathrm{D}$-corrugation (the last close flux surface is moved inward by $\sim 3 \mathrm{~cm}$ at the midplane for a zero poloidal angle). The pedestal position follows the same inward movement, accompanied by a small degradation of the pedestal (relaxation of the density and temperature gradients).

Last, the radial electric field evolution and the braking of the electron perpendicular velocity is observed, following the same trend as in the experiments. Note that to further study the evolution of the plasma rotation in presence of RMPs, the physics of the toroidal momentum damping by the neoclassical toroidal viscosity (NTV) should be taken into account [45, 46]. To do it properly, it would be necessary to calculate the NTV with a kinetic approach by using as input the magnetic field perturbation which takes into account the plasma response; the coupling between the kinetic calculation of the NTV with the MHD calculation of the penetration of the RMPs in JOREK would give a self-consistent approach. This might be done in a future work.

The present work represents an initial stage of this study. In particular our present and future works are mainly focused on the interaction of RMPs with ELMs in realistic experimental cases.
VII. APPENDIX. LINEARIZATION OF THE OHM'S LAW: NON-SCREENING OF THE RMPS WHEN ELECTRON PERPENDICULAR VELOCITY CANCELS

It was found that the cancellation of the perpendicular velocity (18) on the resonant surfaces implies the vanishing of the screening currents, which is the "no screening" condition for RMPs [12]. This can be shown by linearizing the Ohm's law (9) using the Fourier transform $A\left(\psi_{0,0}, \theta, \varphi\right)=A_{0,0}\left(\psi_{0,0}\right)+A_{m, n}\left(\psi_{0,0}\right) e^{i(m \theta+n \varphi)}$ of the variables $P, \psi$ and $J . m$ and $n$ are the poloidal and toroidal mode numbers respectively, and $\psi_{0,0}$ is the equilibrium poloidal flux (label of the flux surfaces). We consider a single mode $(m, n)$ developing on the resonant surface $q=m / n$. The system is supposed to be at the steady state, which means that the growth of the mode has arrived at saturation. We also suppose that the density perturbation is low compared to the average value: $\rho \sim \rho_{0,0}$. In the framework of our model, we assume that electron and ion temperature are equal but the following calculation remains true if $T_{i} \neq T_{e}$. At the first order, equation (9) yields:

$$
\begin{aligned}
& 0=\frac{F_{0}}{R^{2}} i n\left(-u_{m, n}+\frac{\tau_{I C}}{\rho_{0,0}} P_{m, n}\right)+\frac{i m}{R} \psi_{m, n}\left(-\partial_{\psi} u_{0,0}\right. \\
& \left.+\frac{\tau_{I C}}{\rho_{0,0}} \partial_{\psi} P_{0,0}\right)-\frac{i m}{R} \nabla \psi_{0,0}\left(-u_{m, n}+\frac{\tau_{I C}}{\rho_{0,0}} P_{m, n}\right)+\frac{\eta}{R^{2}} J_{m, n}
\end{aligned}
$$

Using the definition of the safety factor $q=\frac{\vec{B} \cdot \nabla \varphi}{\vec{B} \cdot \nabla \theta}=$ $\frac{F_{0} / R^{2}}{|\nabla \psi| / R}=\frac{F_{0}}{R|\nabla \psi|}$, equation (15) becomes:

$$
\begin{aligned}
& \frac{i F_{0}}{R^{2}}\left(\frac{\tau_{I C}}{\rho_{0,0}} P_{m, n}-u_{m, n}\right)\left(\frac{m}{q}-n\right)+\frac{i m}{R} \psi_{m, n}\left(\frac{\tau_{I C}}{\rho_{0,0}} \partial_{\psi} P_{0,0}\right. \\
& \left.-\partial_{\psi} u_{0,0}\right)=\frac{\eta}{R^{2}} J_{m, n}
\end{aligned}
$$

On the resonant surface, $q=m / n$, which implies:

$$
\frac{i m}{R} \psi_{m, n}\left(\frac{\tau_{I C}}{\rho_{0,0}} \partial_{\psi} P_{0,0}-\partial_{\psi} u_{0,0}\right)=\frac{\eta}{R^{2}} J_{m, n}
$$

We can write the perpendicular component of the electron velocity:

$$
\begin{aligned}
V_{\perp, e} & =\left(\vec{V}_{e}^{*}+\vec{V}_{E \times B}\right) \cdot \vec{e}_{\theta} \\
& =\left(\frac{R^{2} \tau_{I C}}{\rho} \nabla P \times \nabla \varphi-R^{2} \nabla u \times \nabla \varphi\right) \cdot \vec{e}_{\theta} \\
& =R\left(\frac{\tau_{I C}}{\rho} \nabla_{\perp} P-\nabla_{\perp} u\right) \cdot \frac{\nabla \psi}{|\nabla \psi|} \\
& =R\left(\frac{\tau_{I C}}{\rho_{0,0}} \partial_{\psi} P_{0,0}-\partial_{\psi} u_{0,0}\right)
\end{aligned}
$$

The combination of the equations (18) and (17) yields:

$$
i m V_{\perp, e} \psi_{m, n}=\eta J_{m, n}
$$


This relation shows that the current perturbation which appears on the resonant surface $q=m / n$ in response to the magnetic perturbation is proportional to the local electron perpendicular velocity $V_{\perp, e}$. Therefore it appears that the cancellation of the perpendicular flow on a resonant surface is the non-screening condition of the corresponding RMP harmonics $(m, n)$ by the plasma rotation. The surfaces where this condition is fulfilled can be found via a $q_{95}$-scan, which shifts the radial position of the resonant surfaces.

\section{Acknowledgments}

This work, supported by the European Communities under the contract of Association between EURATOM and CEA, was carried out within the framework of the European Fusion Development Agreement. The views and opinions expressed herein do not necessarily reflect those of the European Commission nor those of the ITER Organization. This work has benefited from financial support from the National French Research Program (ANR): ANEMOS (2011) and E2T2(2010). The work of Pavel Cahyna was funded by the Grant Agency of the Czech Republic under grant P205/11/2341. Modeling was mainly done on the supercomputers HPC-FF (Julich, Germany), JADE (CINES, France), HELIOS (IFERC, Japan) and CCAMU (Marseille, France). One of the authors (F.Orain) acknowledges Thomas Cartier-Michaud for fruitful discussions.

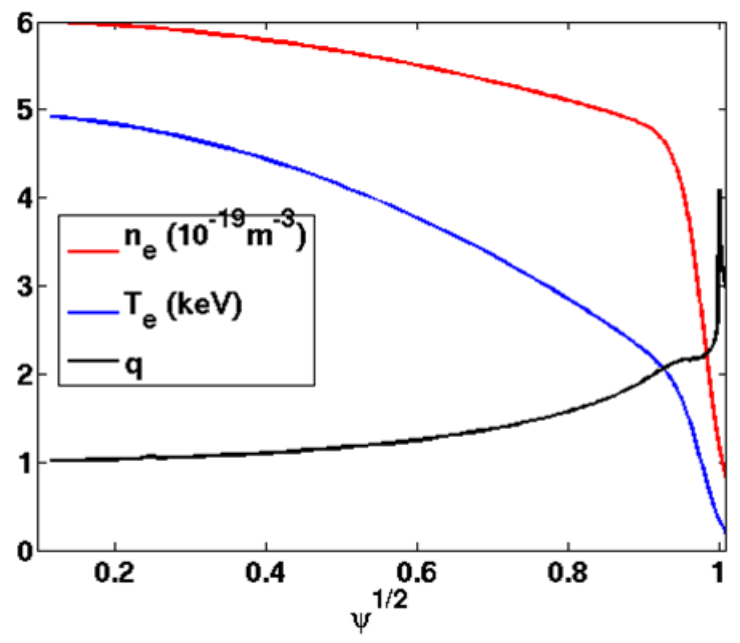

FIG. 1: Initial radial profiles at $t=0$ for JET-like simulations: electron temperature $T_{e}$ in $\mathrm{keV}$, density $n_{e}$ in $10^{19} \mathrm{~m}^{-3}$ and safety factor $q$. These profiles are typical experimental JET profiles (previously used in simulations in [17]); the q-profile is almost flat in the pedestal due to the bootstrap current.

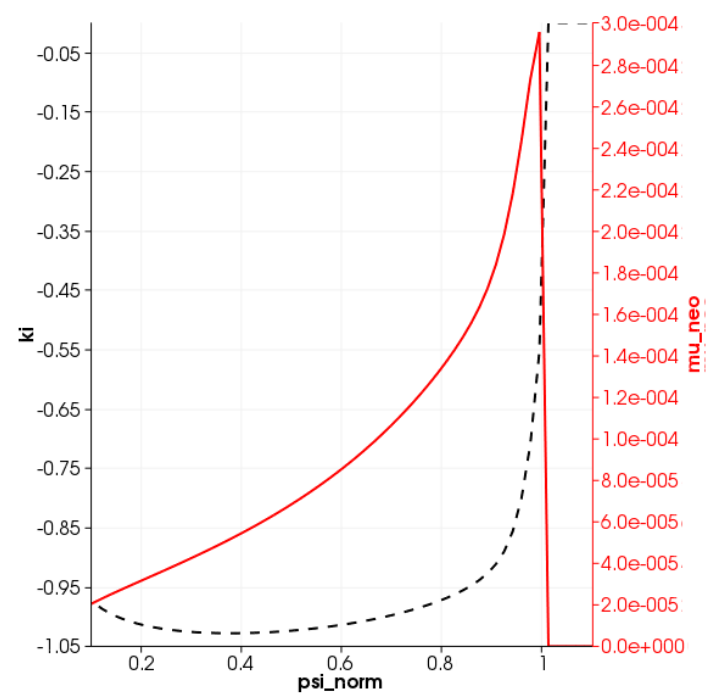

FIG. 2: Realistic radial profiles of the neoclassical coefficients $k_{i}$ (left axis) and $\mu_{i, n e o}$ (right axis) used for simulations in the JET case.

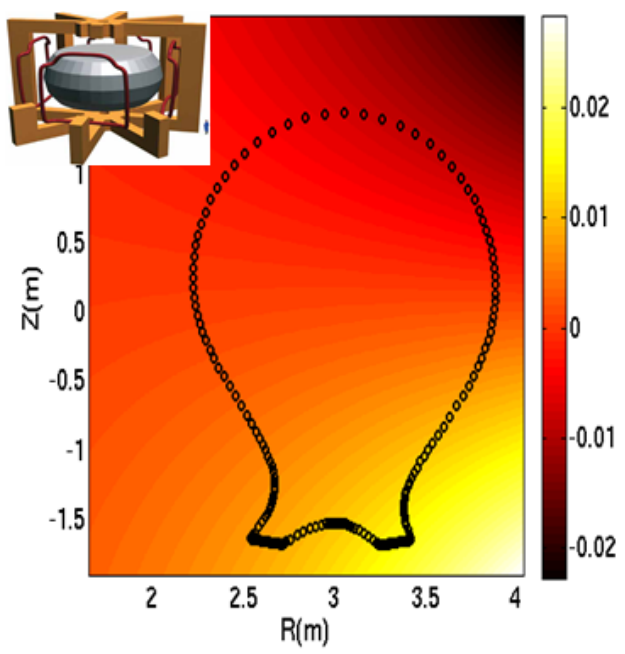

FIG. 3: cosine component of the $n=2$ magnetic flux perturbation generated by EFCC for $I_{\text {coil }}=40 k A t$. The black dots correspond to the boundary of the computational domain where the perturbations are applied. 


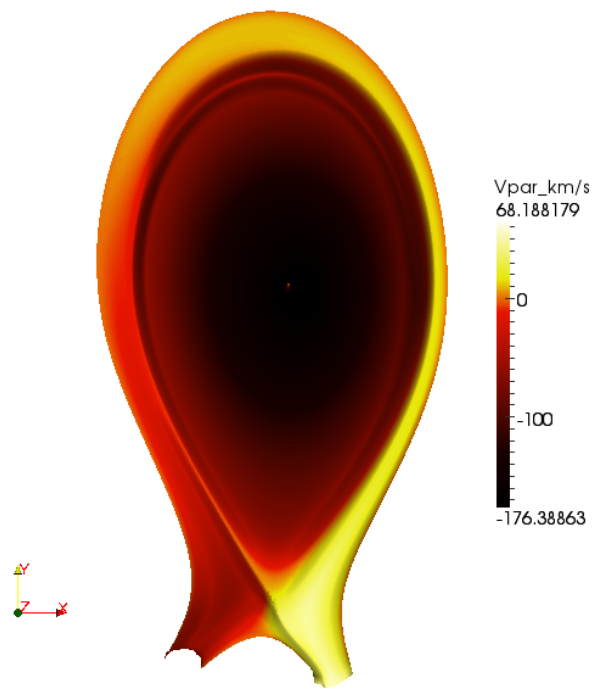

FIG. 4: Parallel velocity (in $\mathrm{km} / \mathrm{s}$ ) in the JET case

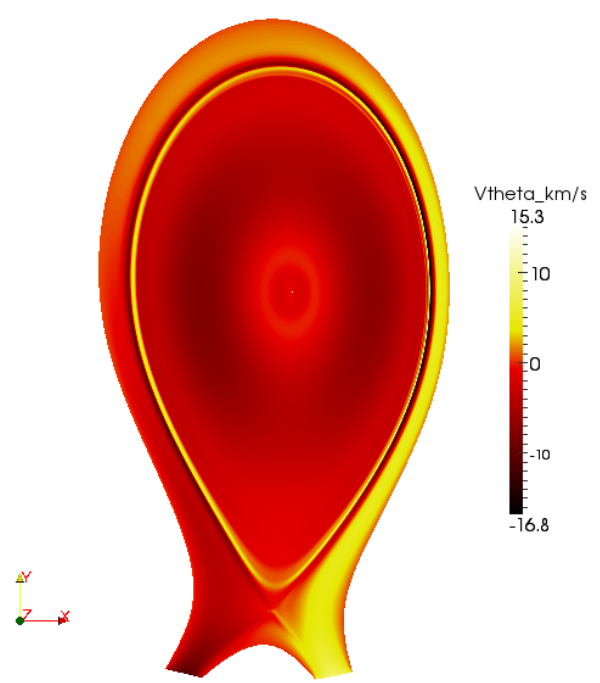

FIG. 5: Poloidal velocity (in $\mathrm{km} / \mathrm{s}$ ) in JET

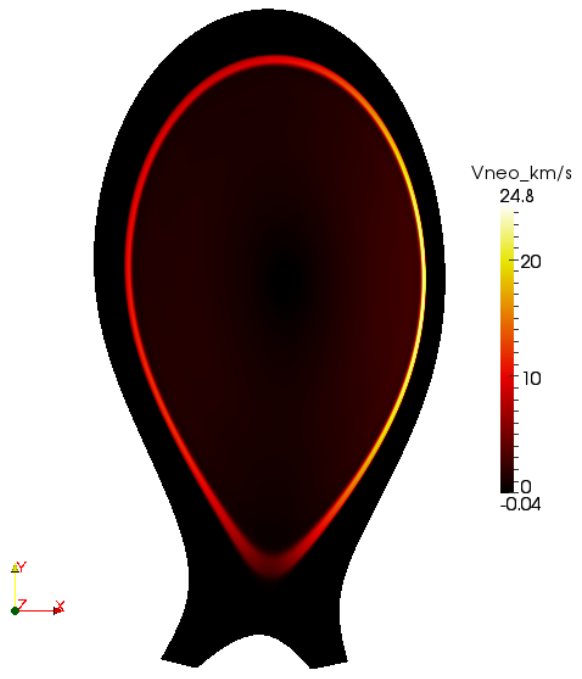

FIG. 6: Neoclassical velocity (in $\mathrm{km} / \mathrm{s}$ ) in JET

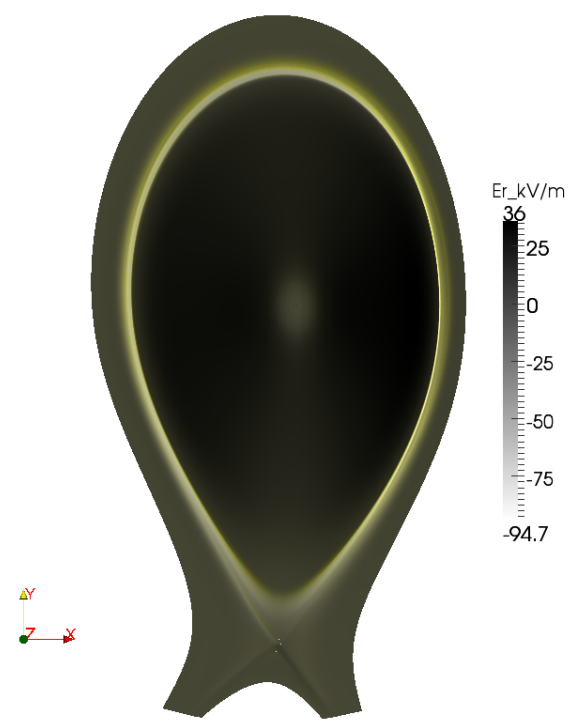

FIG. 7: Radial electric field (in $\mathrm{kV} / \mathrm{m}$ ) in JET with diamagnetic effects, neoclassical effects and the source of toroidal rotation 


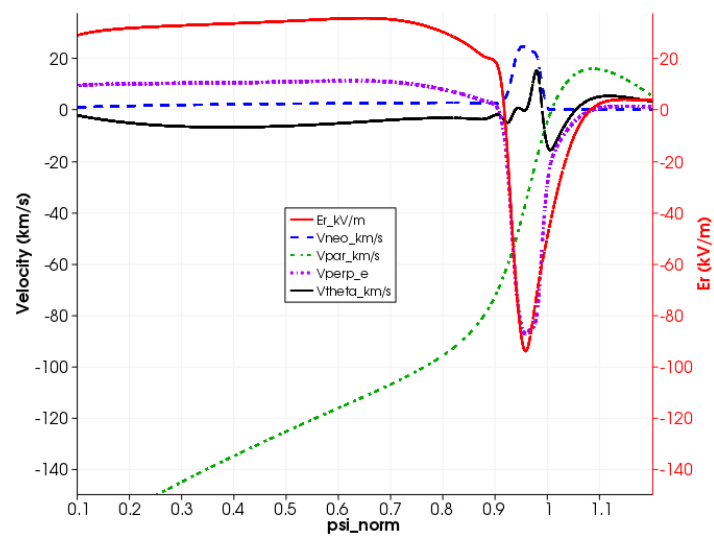

FIG. 8: Radial profiles of the parallel, poloidal and neoclassical velocities and radial electric field at the Low Field Side at the midplane

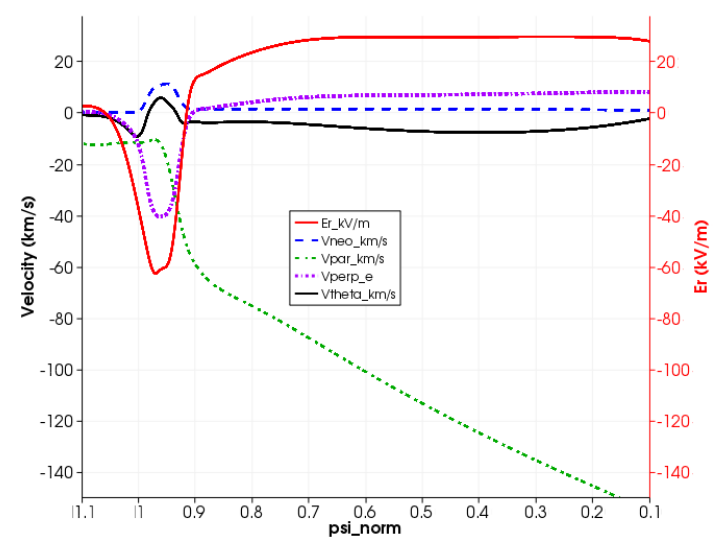

FIG. 9: Radial profiles of the parallel, poloidal and neoclassical velocities and radial electric field at the High Field Side at the midplane

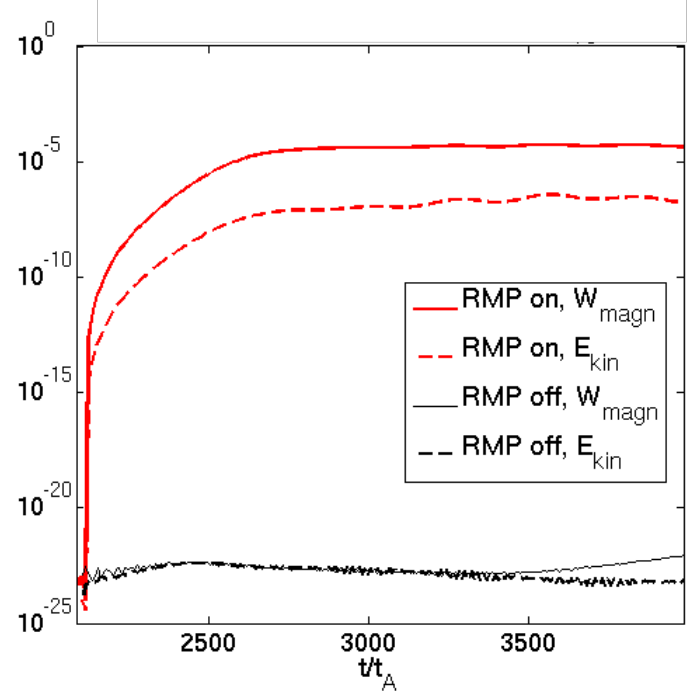

FIG. 10: Magnetic and kinetic energy of the mode $n=2$ without/with RMPs, at high resistivity $\eta_{0}=10^{-7}$ and slow diamagnetic rotation $\left(\tau_{I C}=10^{-3}\right)$

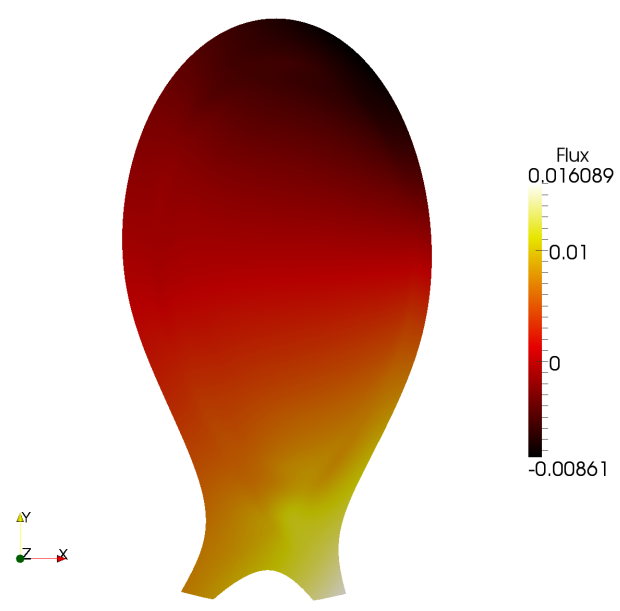

FIG. 11: $n=2$ magnetic flux perturbation $\psi_{n=2}$ penetrating in the plasma $\left(\eta_{0}=10^{-7}, \tau_{I C}=10^{-3}\right)$

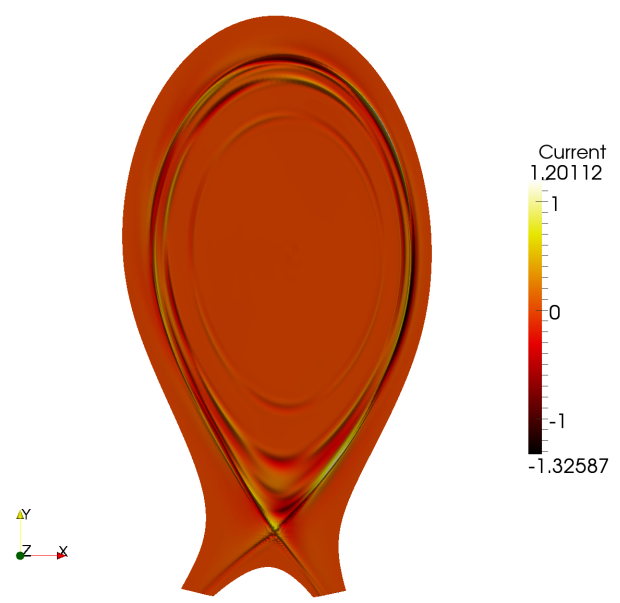

FIG. 12: Response toroidal current perturbation $J_{n=2}$ on the resonant surfaces due to RMPs $\left(\eta_{0}=10^{-7}, \tau_{I C}=10^{-3}\right)$ 


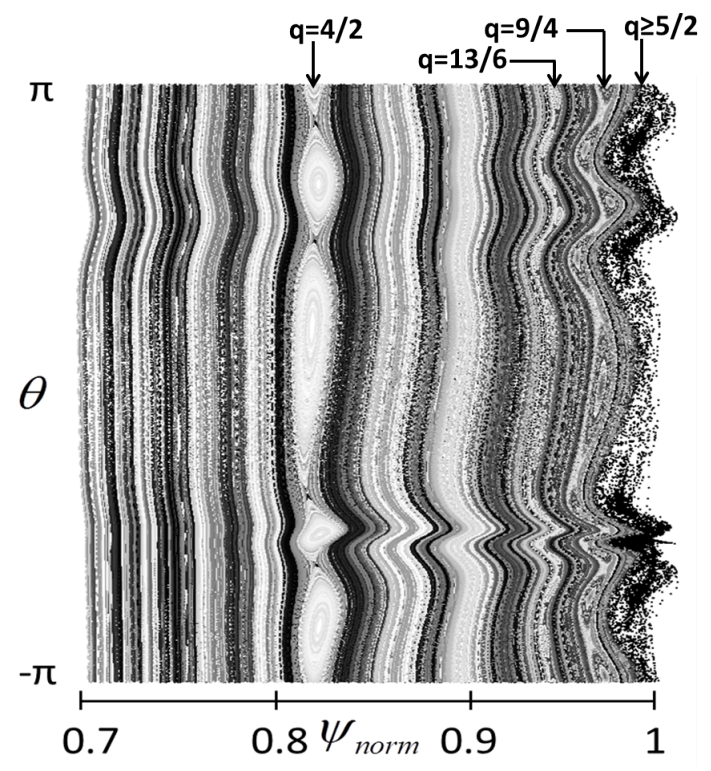

FIG. 13: Poincaré plot of the magnetic topology in $(\psi, \theta)$ coordinates $\left(\eta_{0}=10^{-7}, \tau_{I C}=10^{-3}\right)$

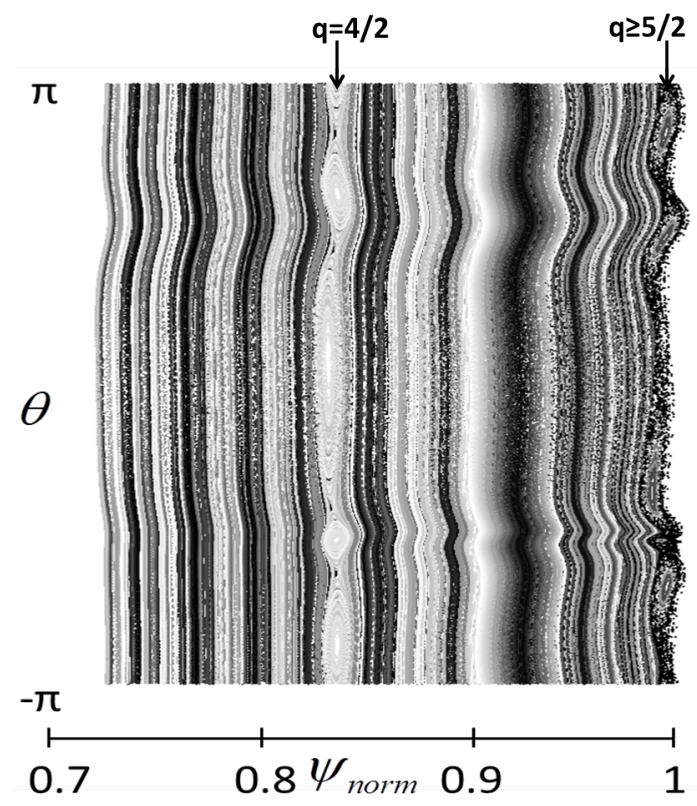

FIG. 14: Poincaré plot of the magnetic topology in $(\psi, \theta)$ coordinates at larger diamagnetic rotation $\tau_{I C}=2 \times 10^{-3}$ (Resistivity is kept $\eta_{0}=10^{-7}$ ). Note that the islands generated by the RMPs are smaller compared to Fig.13, which points out the larger screening induced by the large diamagnetic (poloidal) rotation.

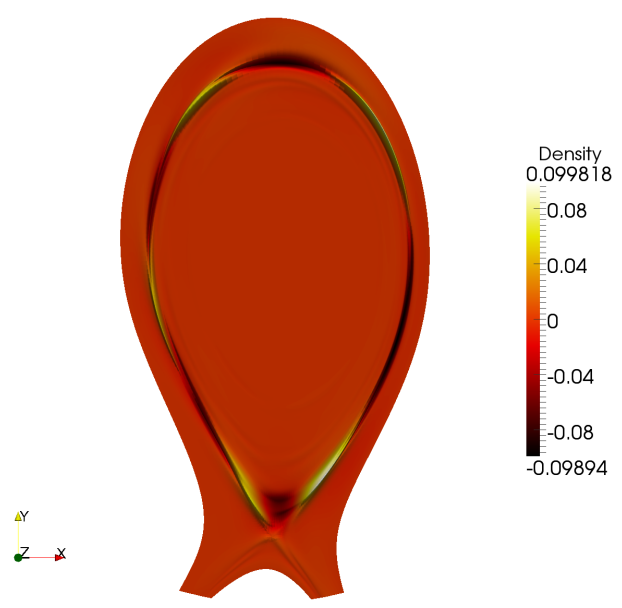

FIG. 15: $n=2$ density perturbation with RMPs, mainly located on the resonant surface $q=5 / 2\left(\eta_{0}=10^{-7}, \tau_{I C}=\right.$ $\left.10^{-3}\right)$

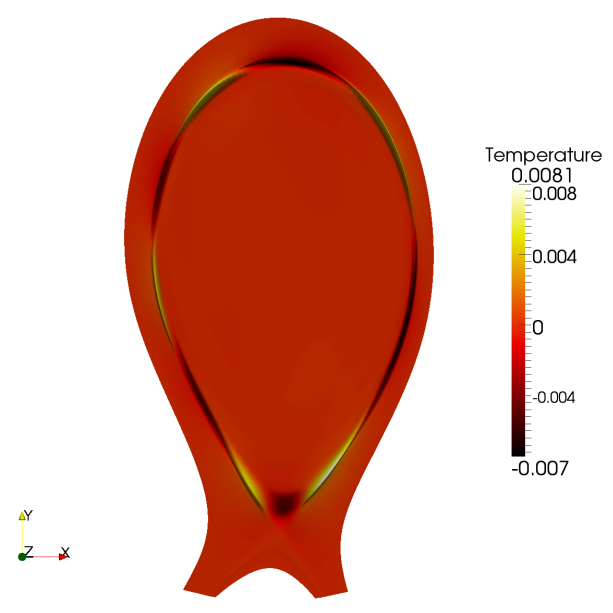

FIG. 16: $n=2$ temperature perturbation with RMPs, mainly located on the resonant surface $q=5 / 2\left(\eta_{0}=10^{-7}, \tau_{I C}=\right.$ $\left.10^{-3}\right)$ 


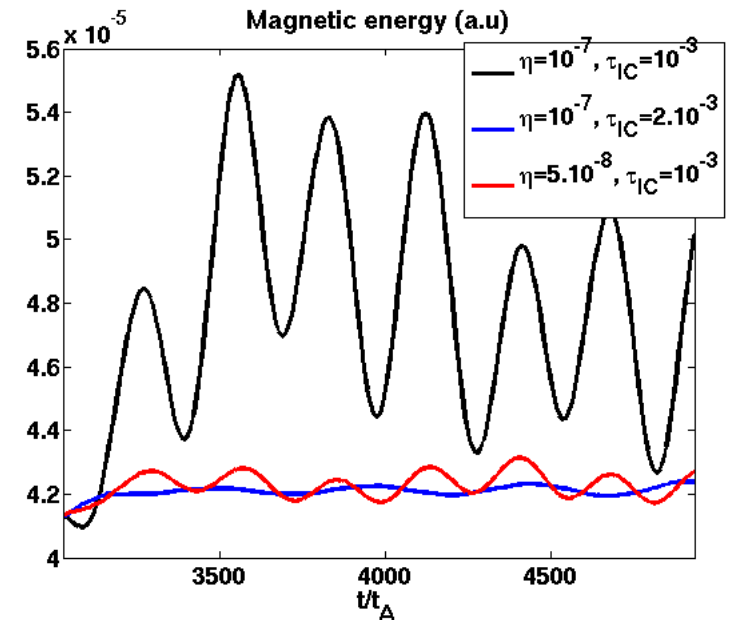

FIG. 17: Three different regimes of penetration of the RMPs: magnetic energy of the mode $n=2$ depending on the diamagnetic parameter $\tau_{I C}$ and the plasma resistivity $\eta_{0}$

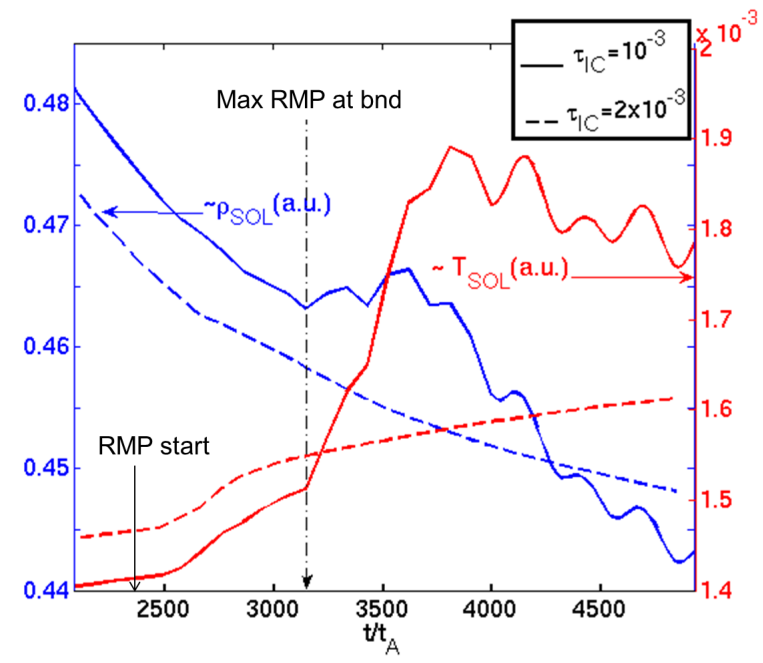

FIG. 18: Volume integrated electron density and temperature in the Scrape-off layer at resistivity $\eta_{0}=10^{-7}$ for two different diamagnetic velocities $\left(\tau_{I C}=10^{-3}\right.$ and $\left.2 \times 10^{-3}\right)$.

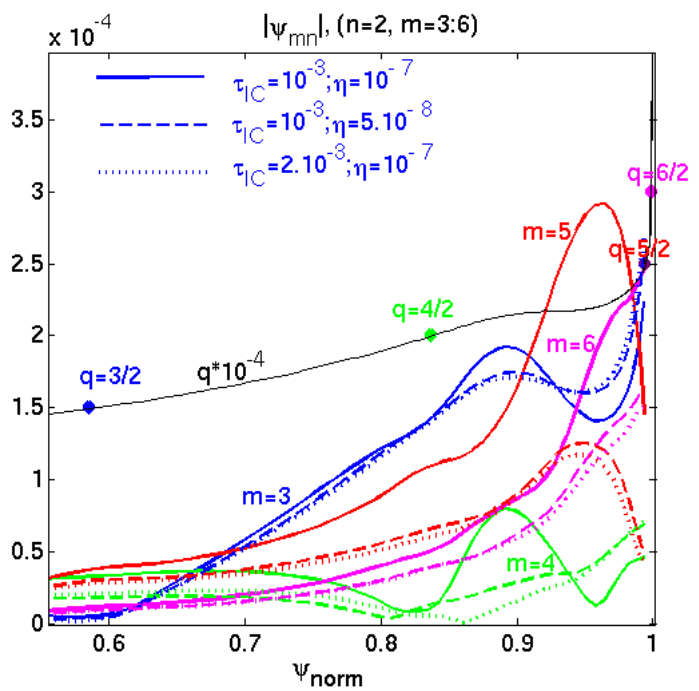

FIG. 19: Fourier harmonics of the magnetic flux $(n=2, m=$ $3-6$ ) depending on the resistivity and the diamagnetic rotation

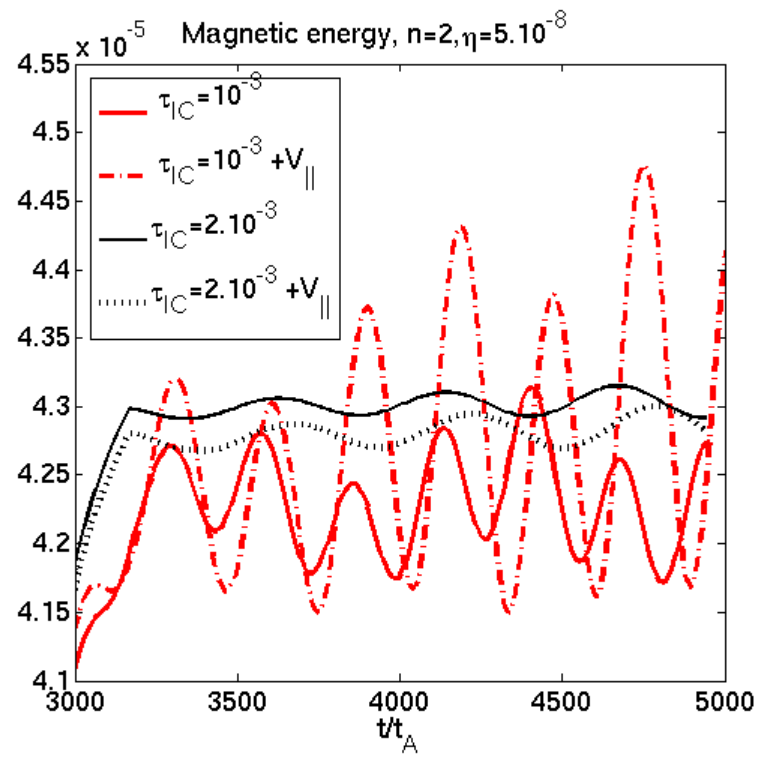

FIG. 20: Effect of the source of parallel rotation on the magnetic energy of the driven mode $n=2$ (resistivity $\eta_{0}=$ $\left.5 \times 10^{-8}\right)$ at low $\left(\tau_{I C}=10^{-3}\right)$ and high $\left(2 \times 10^{-3}\right)$ diamagnetic rotation 


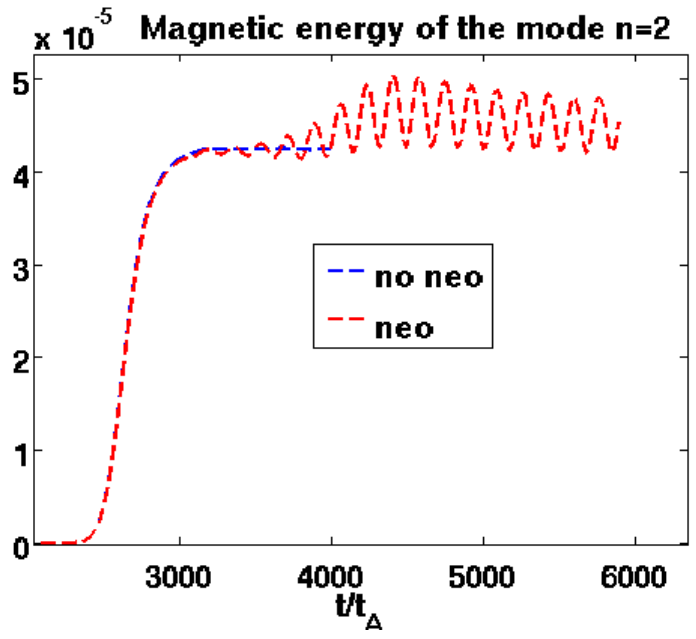

FIG. 21: Effect of the neoclassical friction on the magnetic energy of the driven mode $n=2$ (resistivity $\eta_{0}=10^{-7}$ ) at large diamagnetic rotation $\left(2 \times 10^{-3}\right)$. Without neoclassical effects in the model (in blue), the islands are static. With neoclassical friction included (in red), the generated islands are first static but finally rotate at the ion perpendicular rotation frequency.

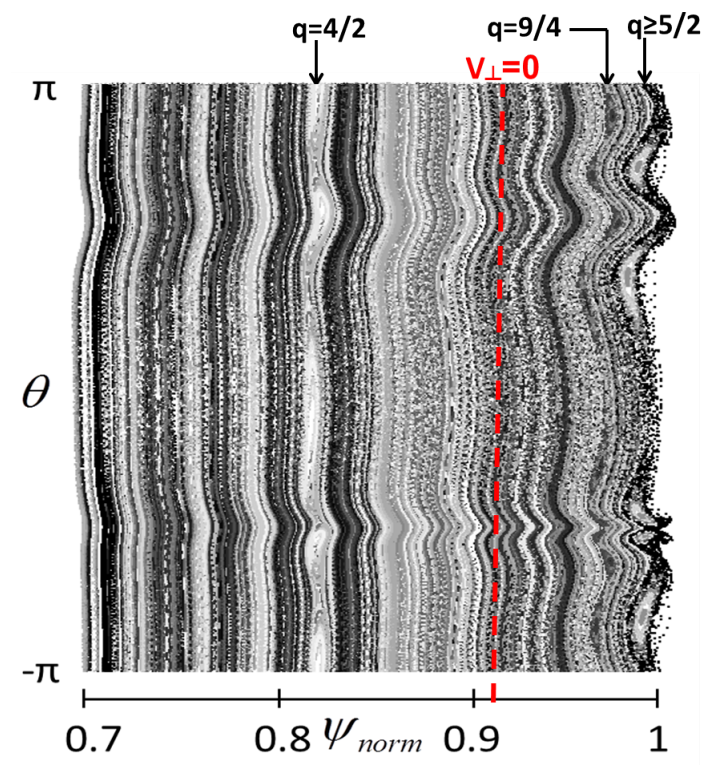

FIG. 22: Poincaré plot of the magnetic topology in $(\psi, \theta)$ coordinates with diamagnetic rotation $\left(\tau_{I C}=2 \times 10^{-3}\right)$, parallel source of rotation and neoclassical friction (resistivity $\left.\eta_{0}=10^{-7}\right)$. The surface where the perpendicular velocity $V_{\perp}$ cancels (indicated by the red dashed line) does not match a resonant surface, which explains that the RMPs do not fully penetrate.

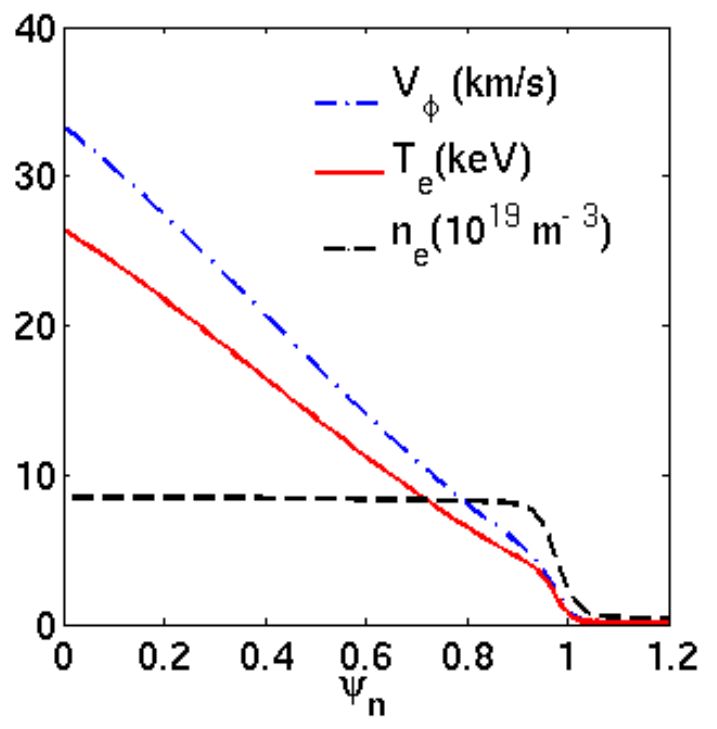

FIG. 23: ITER input density, temperature and toroidal rotation profiles

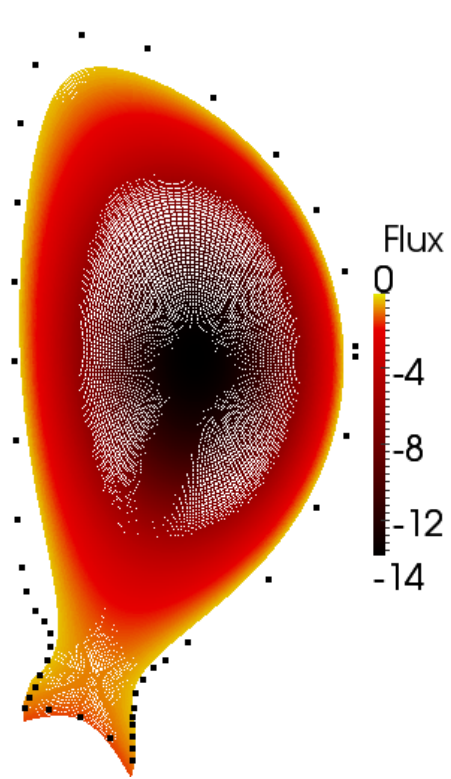

FIG. 24: ITER equilibrium flux-aligned mesh used in modeling 


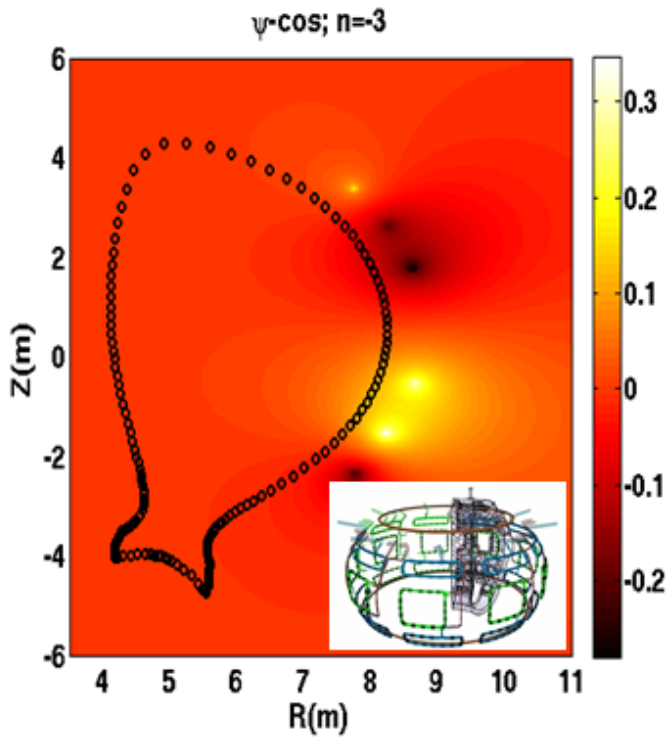

FIG. 25: Vacuum solution for the magnetic flux perturbation $\left(n=3\right.$, IVC at $\left.\max I_{\text {coil }}=54 k A t\right)$ from the ERGOS code, JOREK boundary in black and sketch of the ITER RMP coils in the bottom right corner

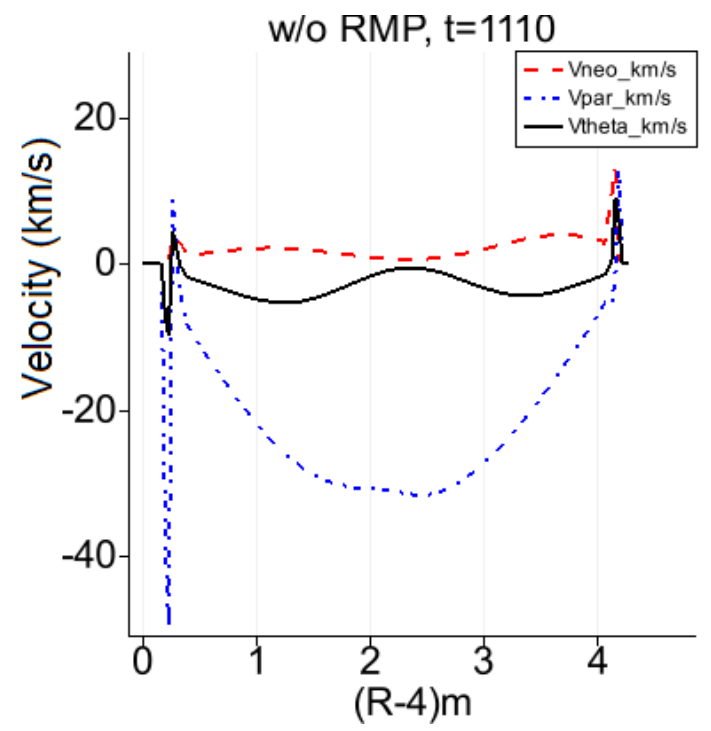

FIG. 26: Profiles of the parallel, poloidal and neoclassical velocities along the midplane in ITER simulations

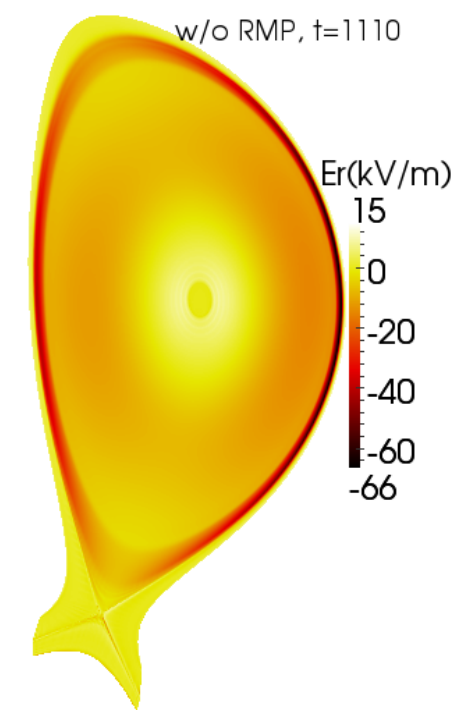

FIG. 27: 2D-profile of the radial equilibrium electric field in ITER (without RMPs)

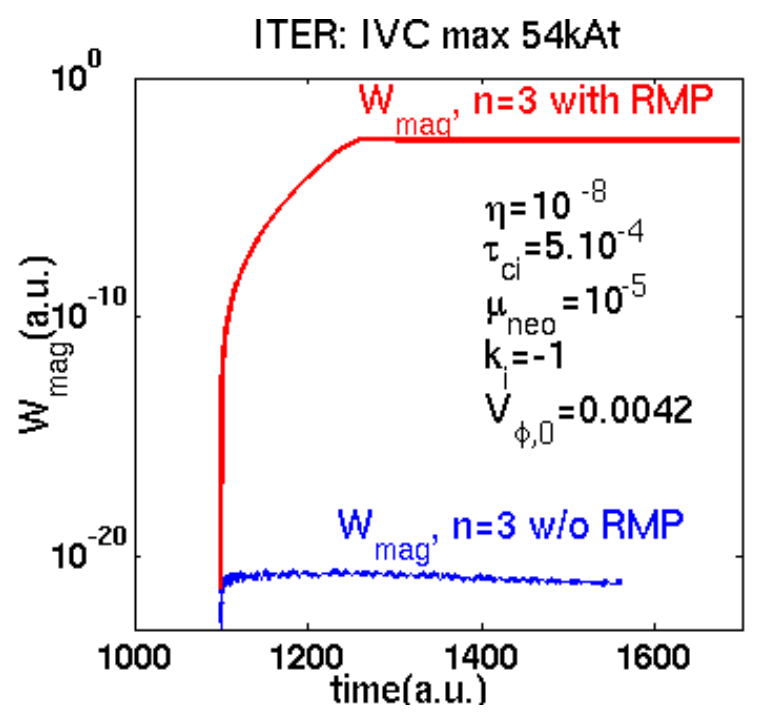

FIG. 28: Magnetic energy of the $n=3$ mode without/with RMPs 


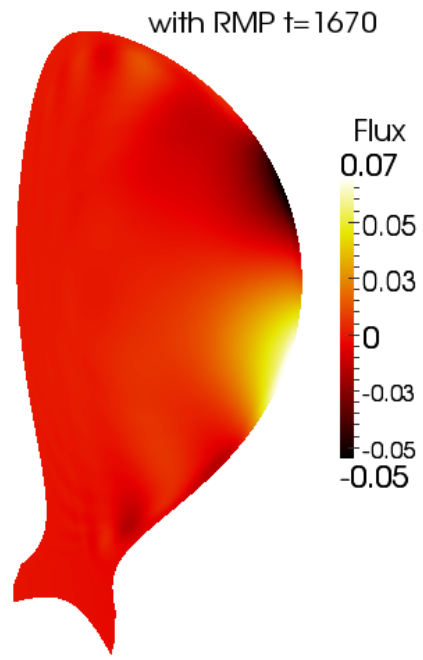

FIG. 29: Static $n=3$ magnetic flux perturbation due to RMPs in ITER

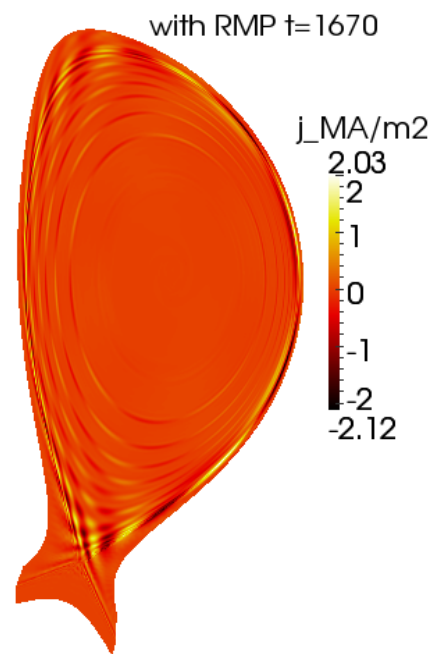

FIG. 30: $n=3$ toroidal current perturbation appearing in response to RMPs in ITER

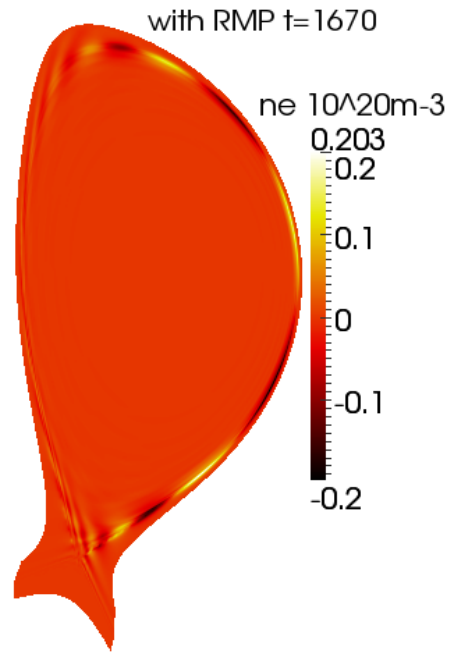

FIG. 31: $n=3$ density perturbation due to RMPs in ITER

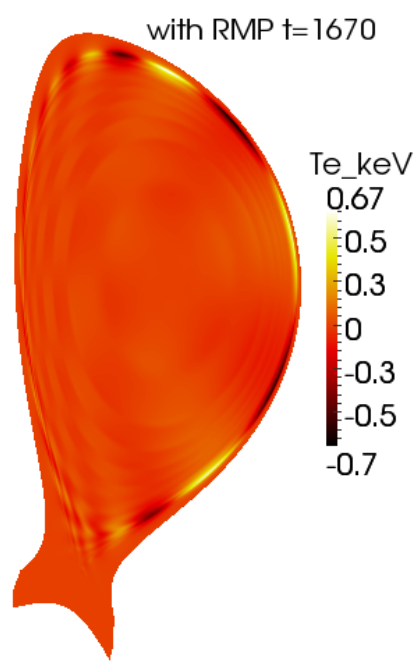

FIG. 32: $n=3$ temperature perturbation due to RMPs in ITER 


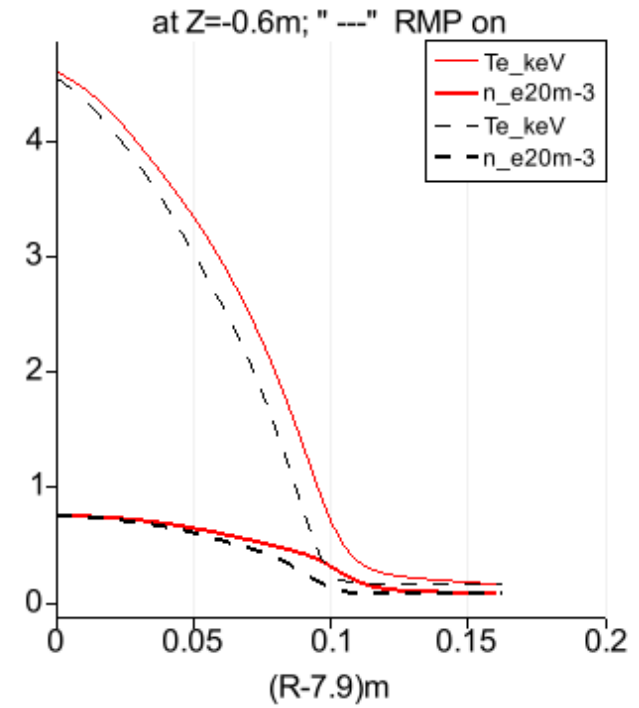

FIG. 33: Density and temperature profiles at the edge for $\mathrm{Z}=-0.6 \mathrm{~m}$ without (full line) and with RMPs (dash)

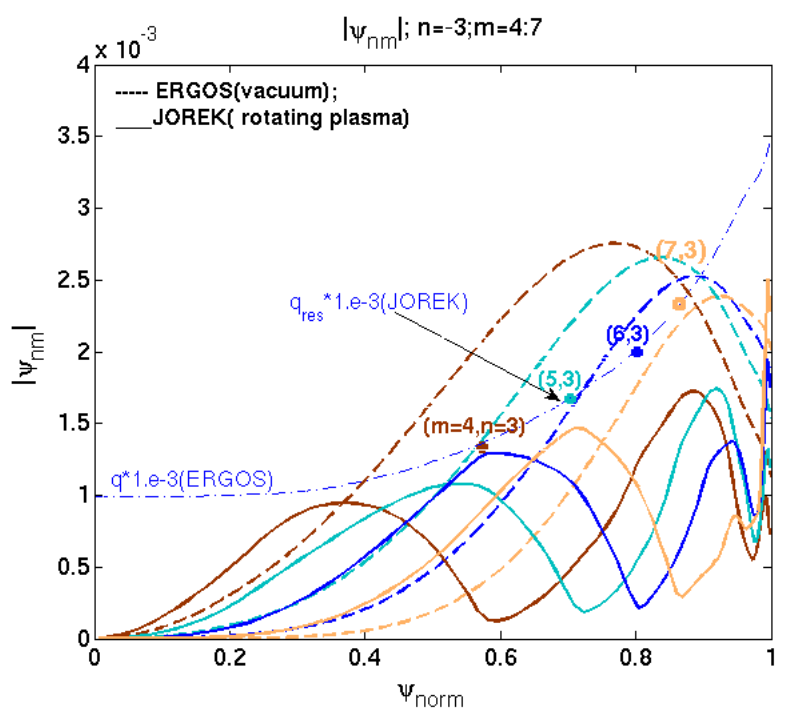

FIG. 34: Fourier harmonics of the magnetic flux perturbation $\left|\psi_{m, n}\right|, n=3, m=4-7$ in the vacuum case (ERGOS code, dash) and taking into account the plasma response (JOREK, full line). The q-profile is given and the positions of the resonant surfaces are indicated by the dots. Note that compared to the vacuum case, all these harmonics $m=4-7$ are screened on the corresponding resonant surfaces $q=m / n$ in presence of the rotating plasma.

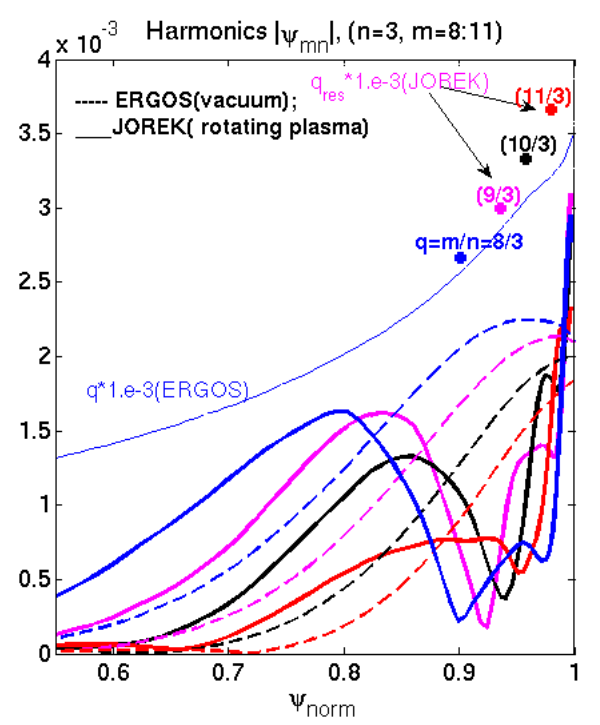

FIG. 35: Fourier harmonics of the magnetic flux perturbation $\left|\psi_{m, n}\right|, n=3, m=8-11$ in the vacuum case (ERGOS code, dash) and taking into account the plasma response (JOREK, full line). The q-profile is given and the positions of the resonant surfaces are indicated by the dots. The edge harmonics $m=10-11$ have a comparable amplitude on the resonant surfaces $q=10-11 / 3$ in the ERGOS and JOREK cases, showing the penetration of these harmonics in the plasma.

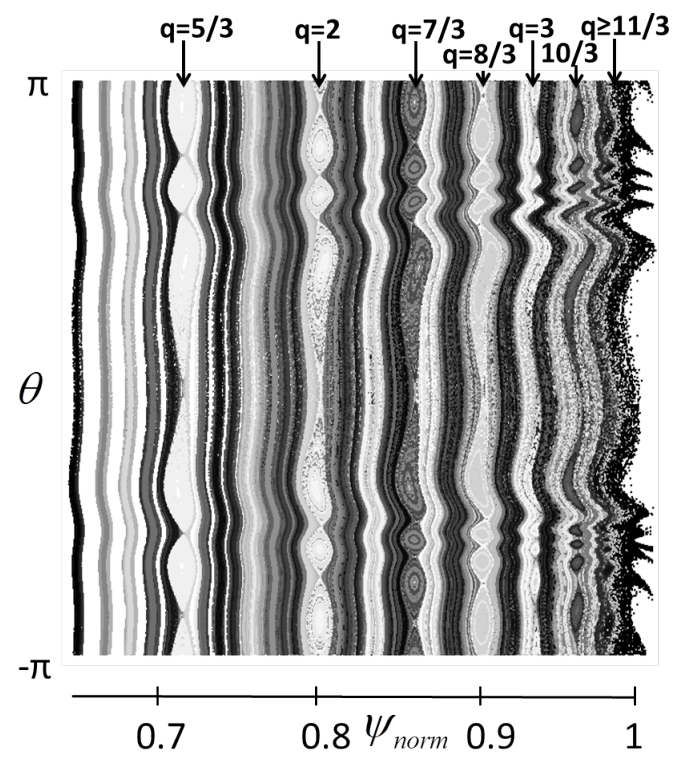

FIG. 36: Poincare plot of the magnetic topology with RMPs in $(\psi, \theta)$ coordinates: magnetic field lines (starting at the edge inside the separatrix) integration after 200 toroidal turns at $\varphi=0$. Resonant surfaces $q=m / 3, m \geq 5$ are indicated. A stochastic layer is formed for $\psi \geq 0.96$ 


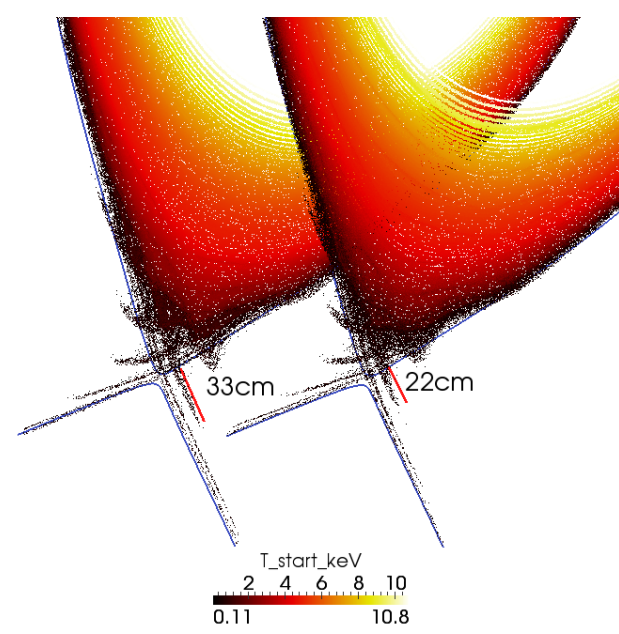

FIG. 37: Magnetic topology near the X-point in $(R, Z)$ coordinates. A lobe structure characteristic of the ergodicity in $\mathrm{X}$-point geometry is observed. On the left, a RMP simulation without neoclassical, diamagnetic flows or toroidal rotation is run for a central resistivity $\eta_{0}=10^{-7}$. On the right, the same RMP simulation is run with all flows included for a central resistivity $\eta_{0}=10^{-8}$ : the resulting lobe size is smaller by $1 / 3$ compared to the the case without flows.

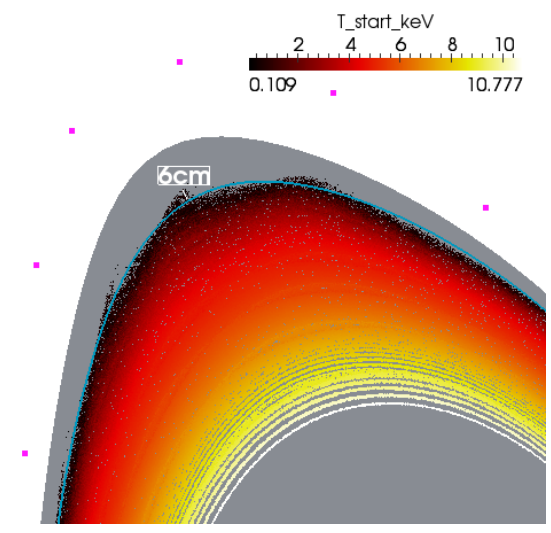

FIG. 38: Magnetic topology at the top of the machine. Small deformations of the separatrix $(\sim 6 \mathrm{~cm})$ are observed.

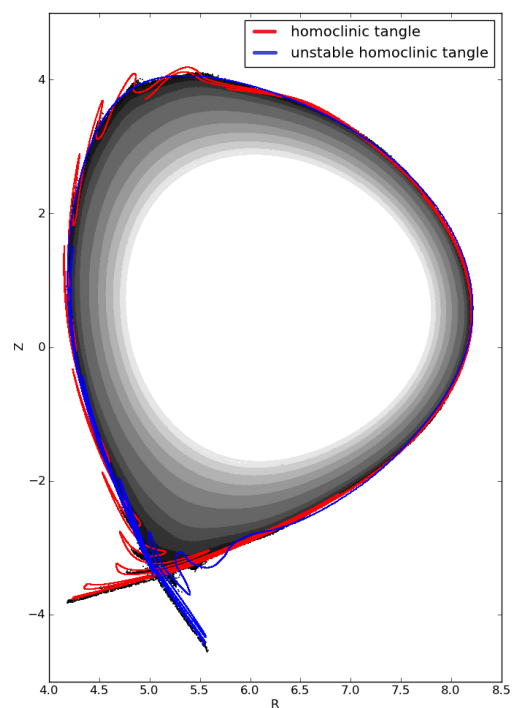

FIG. 39: Superposition of the Poincaré plot of the magnetic topology in $(R, Z)$ coordinates with the stable (red) and unstable (blue) homoclinic tangles defining the deformation of the separatrix.

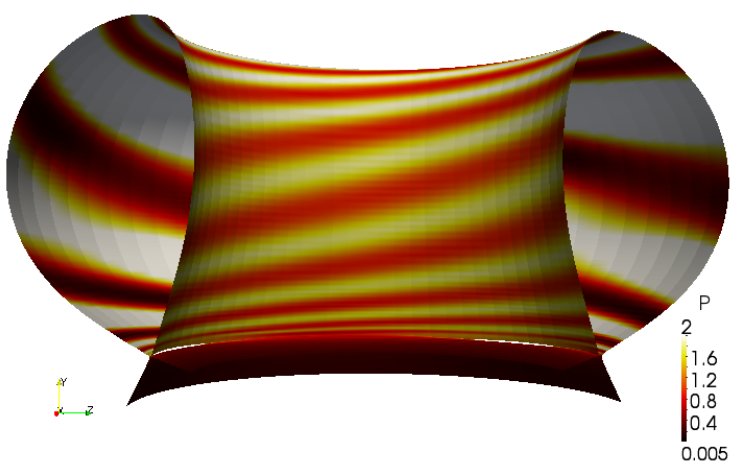

FIG. 40: 3D-plot of the pressure on an initial equilibrium flux surface without RMP near the separatrix. The 3Ddeformation corresponds to a mode $(n=3, m \sim 12)$. 


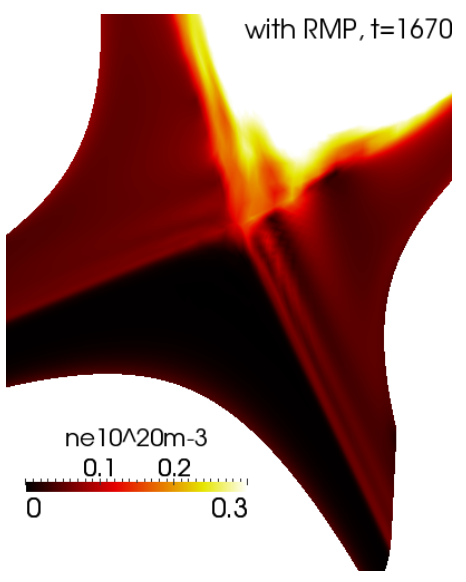

FIG. 41: Degradation of the density profile near the X-point due to RMPs

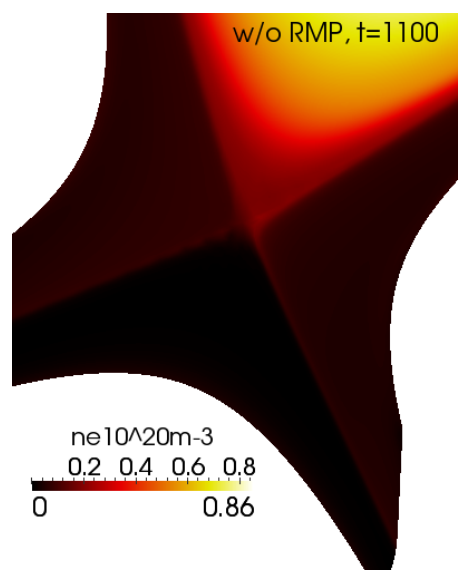

FIG. 42: Density near the X-point without RMPs

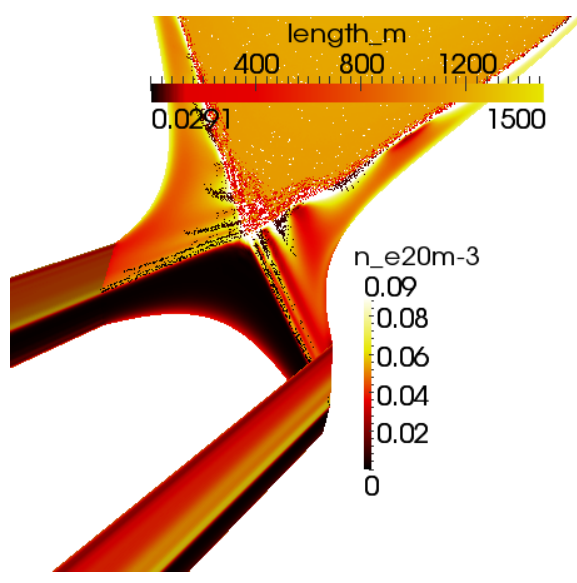

FIG. 43: Superposition of the 2D-plot (in the poloidal plane) of the magnetic topology (connection length) near the Xpoint with the electron density on the divertor targets (in the toroidal direction). Note the induced splitting of the strike points on the outer divertor target.

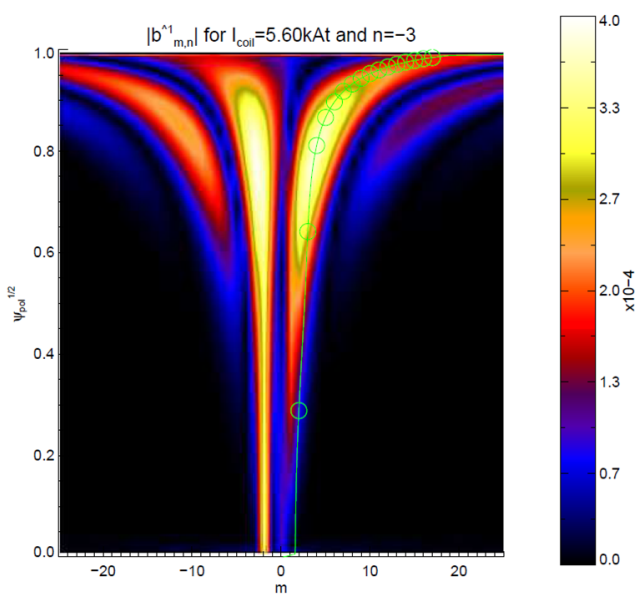

FIG. 44: Fourier harmonics of the radial magnetic field generated by the RMP coils in disposition 90L: in this configuration, the magnetic perturbation is maximal on the resonances $(m, n=3)$

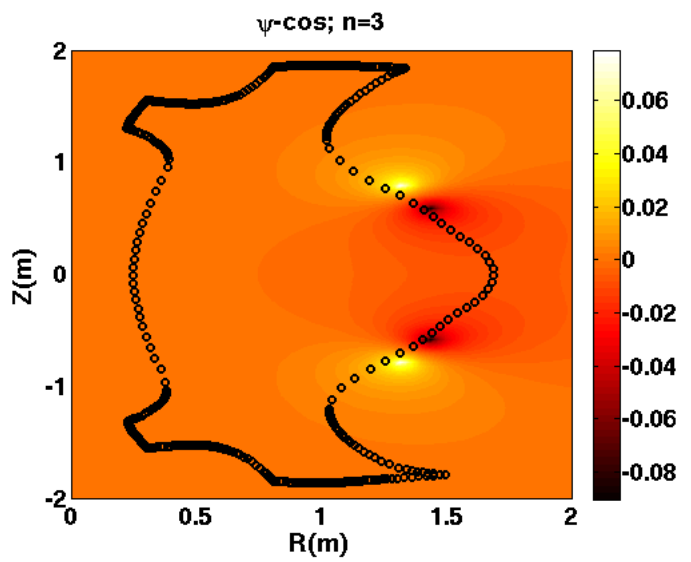

FIG. 45: Input resonant magnetic perturbation generated by the RMP coils calculated in the vacuum with the ERGOS code and applied as boundary conditions of the computational domain (in black)

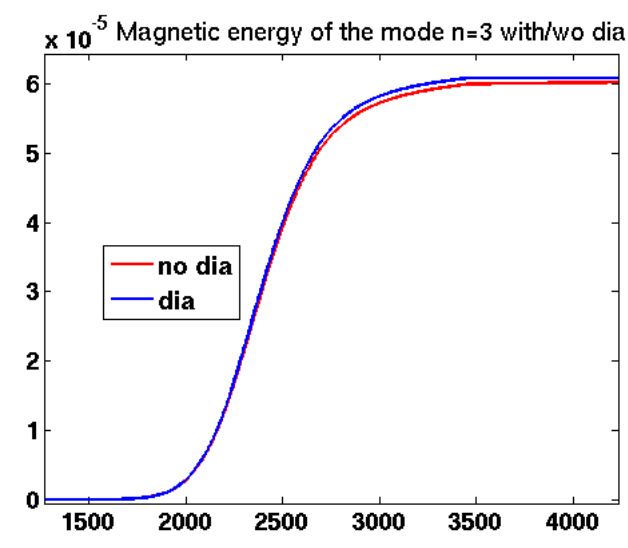

FIG. 46: Magnetic energy of the mode $n=3$ driven by RMPs in the 2 cases: without and with diamagnetic effects 


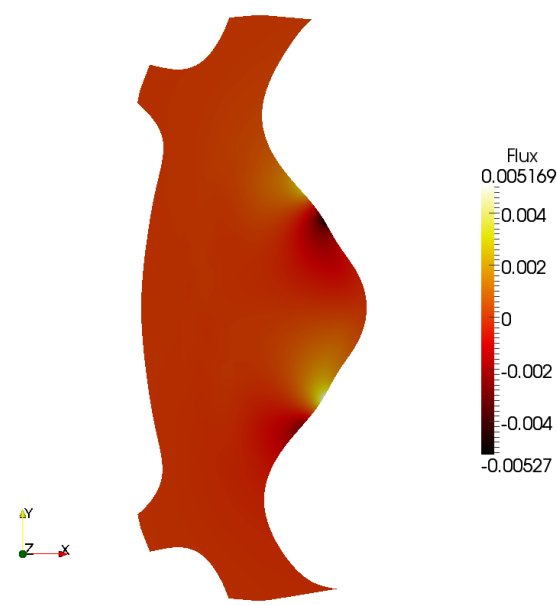

FIG. 47: Magnetic flux perturbation penetrating in the plasma (without diamagnetic effects)

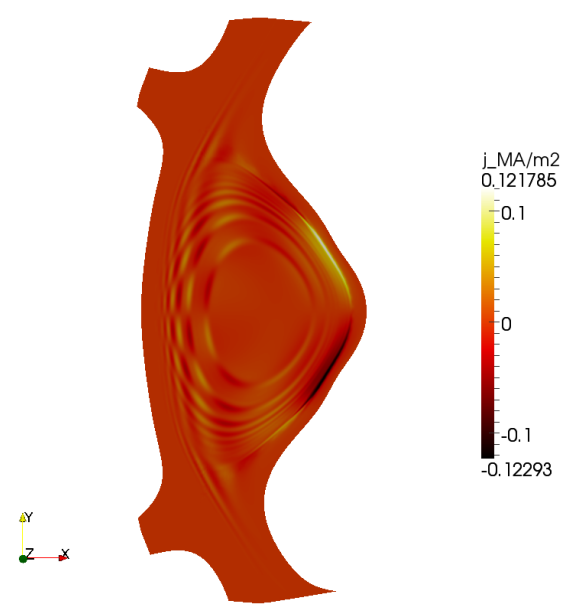

FIG. 48: Current perturbation generated on the resonant surfaces $q=m / n, n=3$ (without diamagnetic effects)

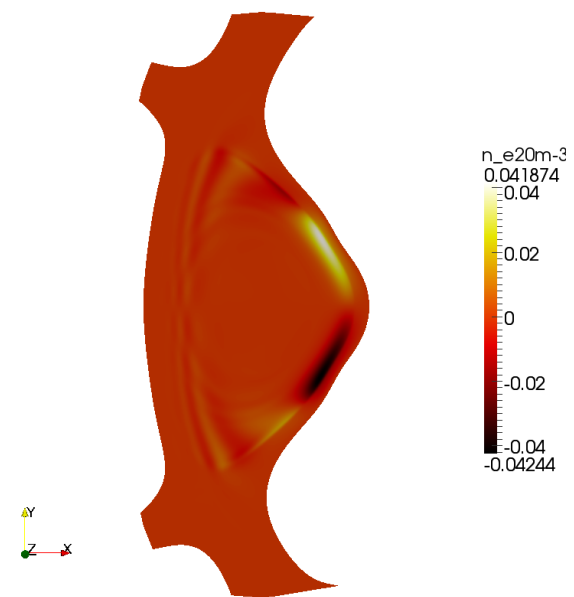

FIG. 49: Density perturbation with $n=3$ RMPs in MAST (without diamagnetic effects)

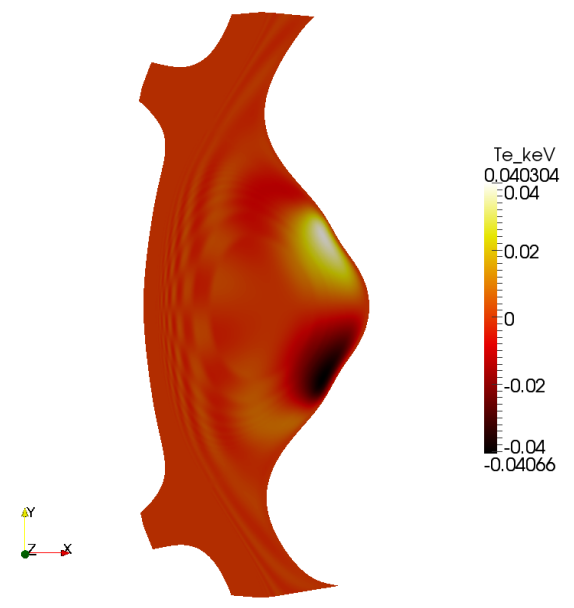

FIG. 50: Electron temperature perturbation with $n=3$ RMPs in MAST (without diamagnetic effects) 


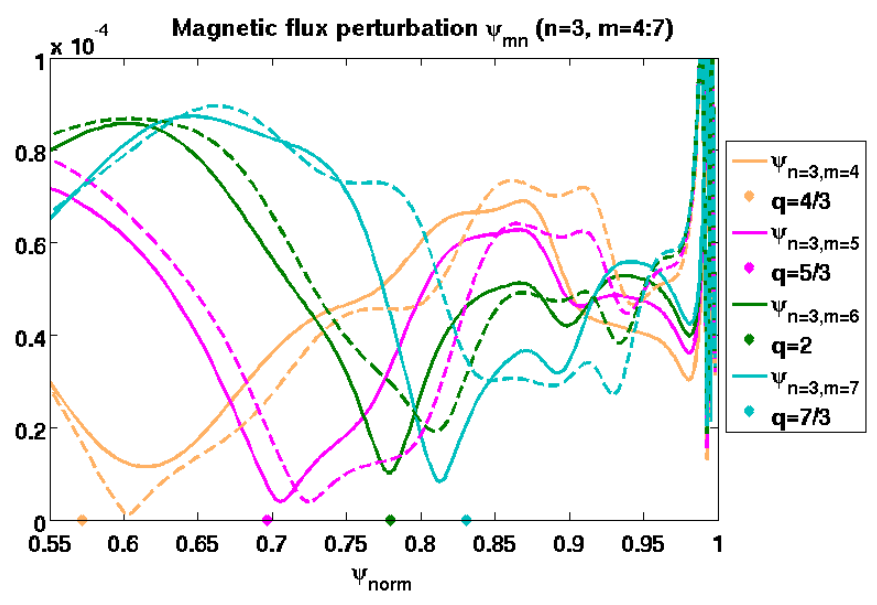

FIG. 51: Radial profile of the Fourier harmonics of the magnetic perturbation $\left|\psi_{m, n}\right|, m=4-7$ in both cases: without diamagnetic effects (dash) and with diamagnetic effects included (full line). The approximate position of the resonant surfaces $q=m / n$ are represented by the dots. All the central harmonics $m=4-7$ are screened near their corresponding resonant surfaces in both cases

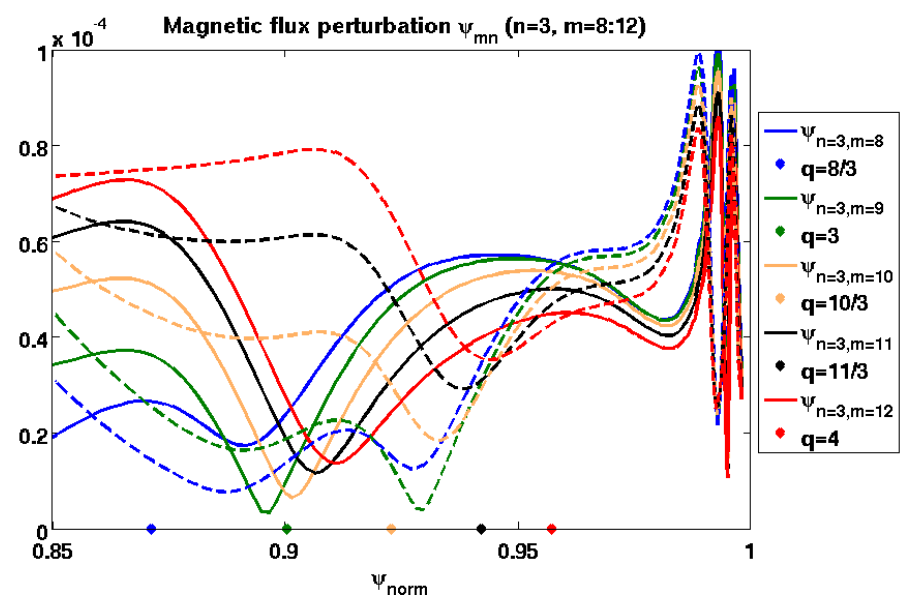

FIG. 52: Radial profile of the Fourier harmonics of the magnetic perturbation $\left|\psi_{m, n}\right|, m=8-12$ in both cases: without diamagnetic effects (dash) and with diamagnetic effects included (full line). The approximate position of the resonant surfaces $q=m / n$ are represented by the dots. The more central harmonics $m=8-9$ are screened near their corresponding resonant surfaces in both cases, whereas the external ones $(m=10-12)$ penetrate close to the surface $q=m / n$ in both cases but are slightly more amplified in the case including the diamagnetic effects.

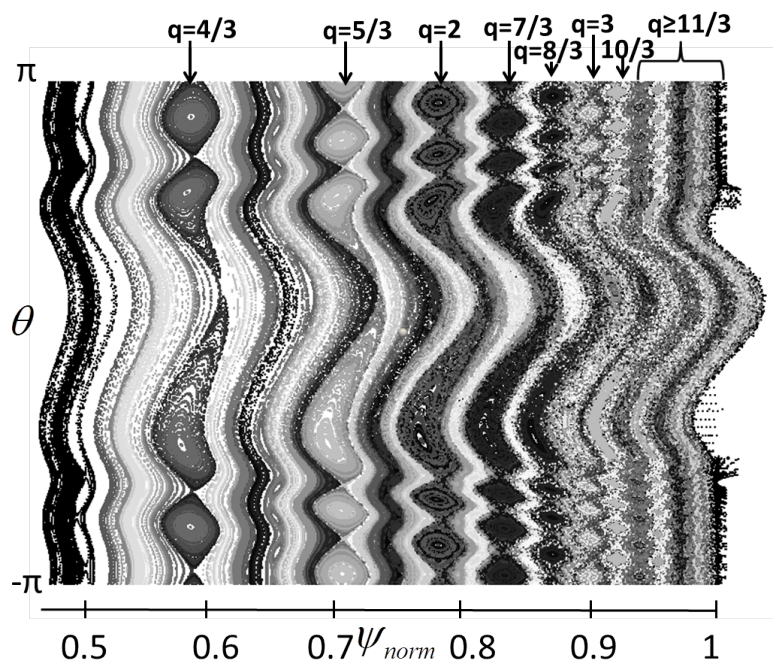

FIG. 53: Poincare plot of the magnetic topology in MAST without diamagnetic rotation, in $(\psi, \theta)$ coordinates: magnetic field lines integration after 200 toroidal turns. The resonant surfaces $q=m / 3, m \geq 4$ are indicated. A stochastic layer is formed for $\psi \geq 0.96$

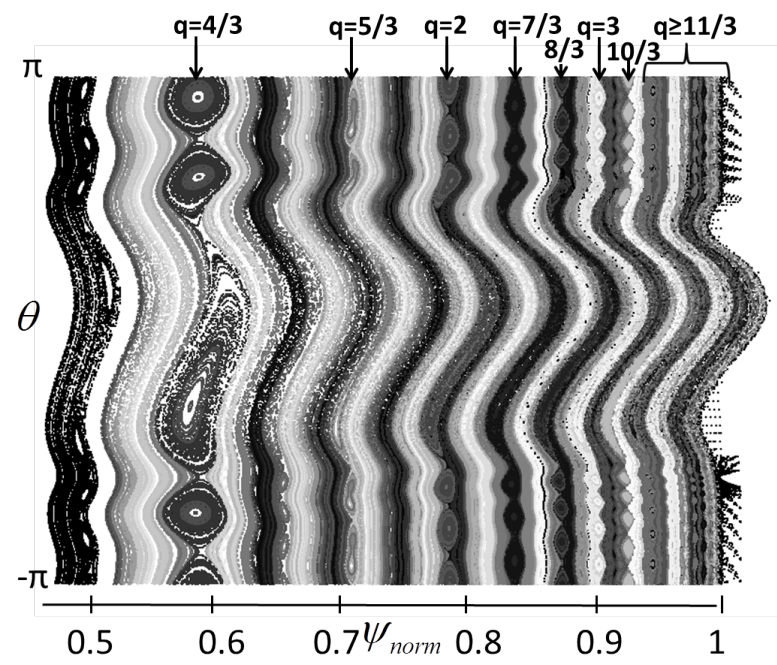

FIG. 54: Poincare plot of the magnetic topology in MAST with diamagnetic rotation included, in $(\psi, \theta)$ coordinates: magnetic field lines integration after 200 toroidal turns. The resonant surfaces $q=m / 3, m \geq 4$ are indicated. A stochastic layer is formed for $\psi \geq 0.96$ 


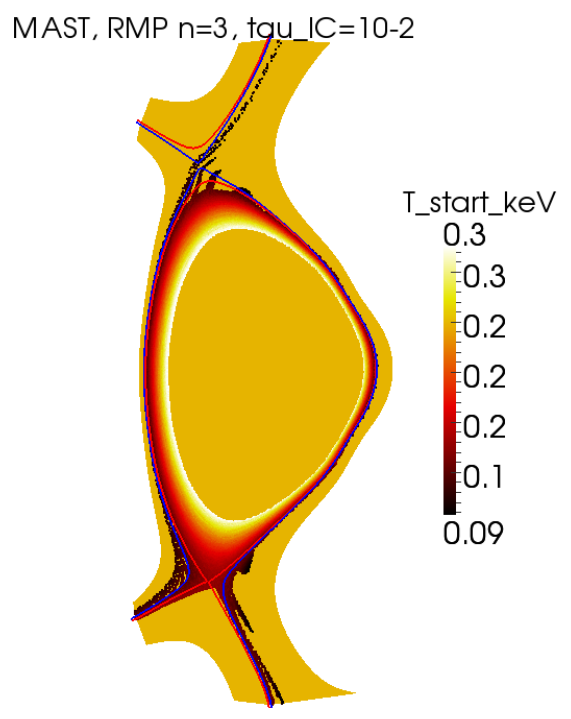

FIG. 55: Poincare plot of the magnetic topology with diamagnetic flow. The color represents the temperature of the starting point of the field line inside the separatrices. The length of the lobes near the X-points is sensibly the same in the case without diamagnetic flow

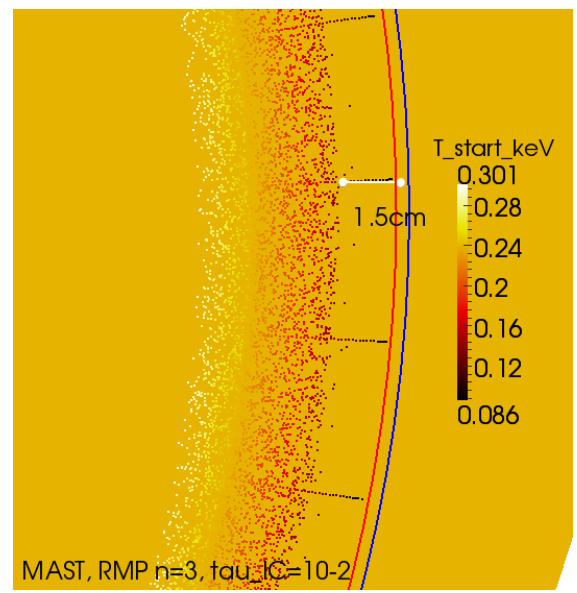

FIG. 56: Zoom in Poincare plot Fig.55 around the midplane (case with diamagnetic flow): the displacement of the last closed flux surface due to RMPs at the toroidal angle $\varphi=0^{\circ}$ is approximately $1.5 \mathrm{~cm}$

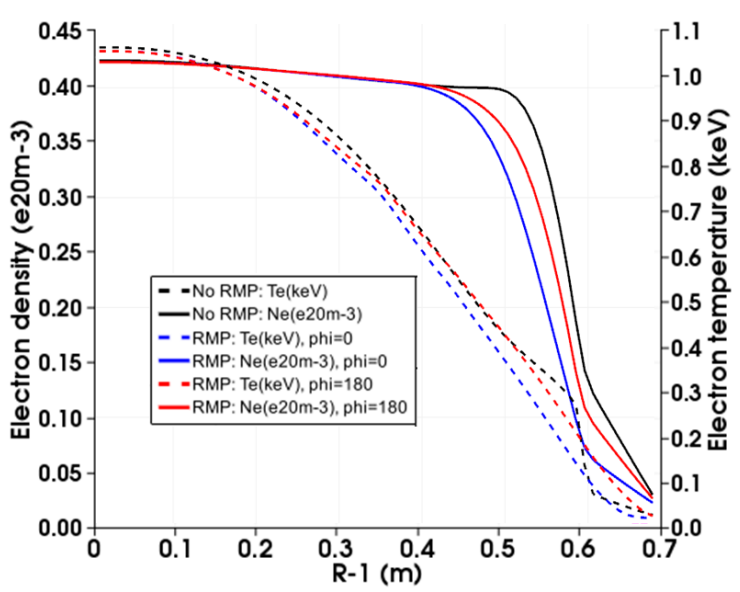

FIG. 57: Radial profiles of the electron temperature (dash) and the density (full line) at the midplane without RMPs (black), with RMPs at the toroidal angles $\varphi=0^{\circ}$ (blue) and $180^{\circ}$ (red): note the small degradation of the pedestal and the $3 \mathrm{D}$-deformation of density and temperature due to RMPs

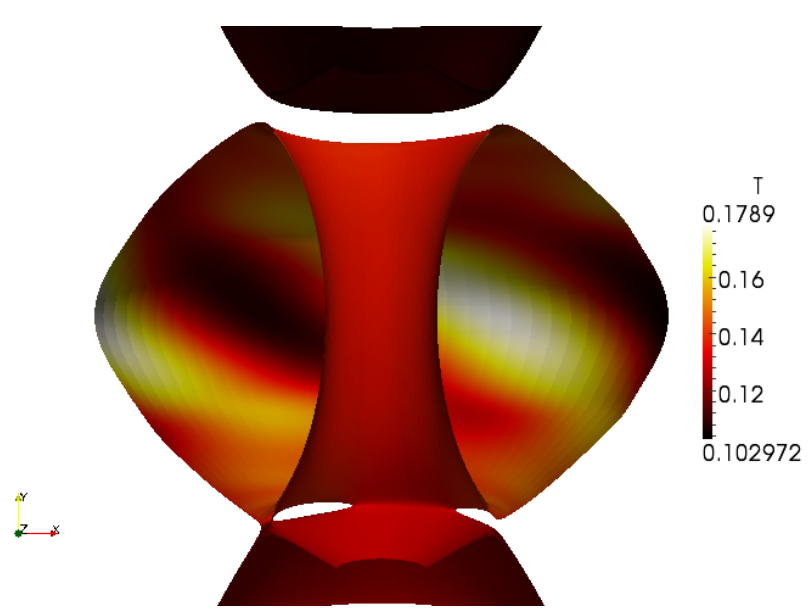

FIG. 58: 3D-deformation of the electron temperature near the separatrix due to RMPs 


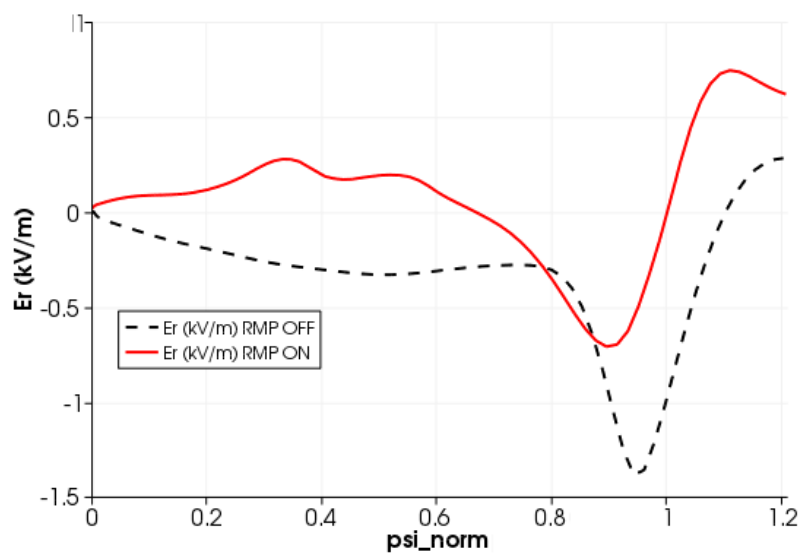

FIG. 59: Radial profile of the radial electric field at the midplane in the LFS without RMPs (- - black) and with RMPs (red)

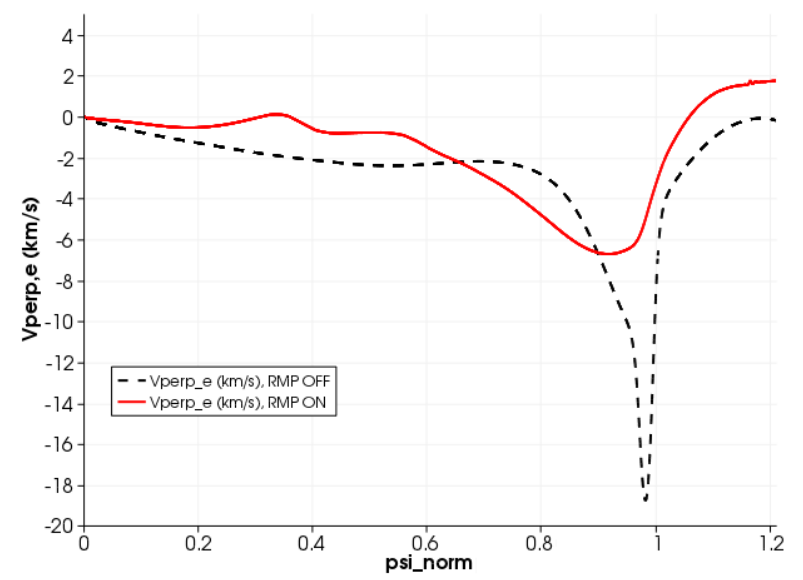

FIG. 60: Radial profile of the electron perpendicular velocity at the midplane in the LFS without RMPs (- - black) and with RMPs (red) 
[1] R.J. Hawryluk, D.J. Campbell, G. Janeschitz, P.R. Thomas, R. Albanese, R. Ambrosino, C. Bachmann, L. Baylor, M. Bécoulet, I. Benfatto, J. Bialek, A. Boozer, A. Brooks, R. Budny, T. Casper, M. Cavinato, J.-J. Cordier, V. Chuyanov, E. Doyle, T. Evans, G. Federici, M. Fenstermacher, H. Fujieda, K. G'al, A. Garofalo, L. Garzotti, D. Gates, Y. Gribov, P. Heitzenroeder, T.C. Hender, N. Holtkamp, D. Humphreys, I. Hutchinson, K. Ioki, J. Johner, G. Johnson, Y. Kamada, A. Kavin, C. Kessel, R. Khayrutdinov, G. Kramer, A. Kukushkin, K. Lackner, I. Landman, P. Lang, Y. Liang, J. Linke, B. Lipschultz, A. Loarte, G.D. Loesser, C. Lowry, T. Luce, V. Lukash, S. Maruyama, M. Mattei, J. Menard, M. Merola, A. Mineev, N. Mitchell, E. Nardon, R. Nazikian, B. Nelson, C. Neumeyer, J.-K. Park, R. Pearce, R.A. Pitts, A. Polevoi, A. Portone, M. Okabayashi, P.H. Rebut, V. Riccardo, J. Roth, S. Sabbagh, G. Saibene, G. Sannazzaro, M. Schaffer, M. Shimada, A. Sen, A. Sips, C.H. Skinner, P. Snyder, R. Stambaugh, E. Strait, M. Sugihara, E. Tsitrone, J. Urano, M. Valovic, M. Wade, J. Wesley, R. White, D.G. Whyte, S. Wu, M. Wykes, and L. Zakharov. Principal physics developments evaluated in the iter design review. Nuclear Fusion, 49(6):065012, 2009.

[2] M. E. Fenstermacher, T. E. Evans, T. H. Osborne, M. J. Schaffer, M. P. Aldan, J. S. deGrassie, P. Gohil, I. Joseph, R. A. Moyer, P. B. Snyder, R. J. Groebner, M. Jakubowski, A. W. Leonard, O. Schmitz, and DIIID Team. Effect of island overlap on edge localized mode suppression by resonant magnetic perturbations in diii-d. Physics of Plasmas, 15(5), MAY 2008.

[3] T.E. Evans, M.E. Fenstermacher, R.A. Moyer, T.H. Osborne, J.G. Watkins, P. Gohil, I. Joseph, M.J. Schaffer, L.R. Baylor, M. Bcoulet, J.A. Boedo, K.H. Burrell, J.S. deGrassie, K.H. Finken, T. Jernigan, M.W. Jakubowski, C.J. Lasnier, M. Lehnen, A.W. Leonard, J. Lonnroth, E. Nardon, V. Parail, O. Schmitz, B. Unterberg, and W.P. West. Rmp elm suppression in diii-d plasmas with iter similar shapes and collisionalities. Nuclear Fusion, 48(2):024002, 2008.

[4] W. Suttrop, T. Eich, J. C. Fuchs, S. Günter, A. Janzer, A. Herrmann, A. Kallenbach, P. T. Lang, T. Lunt, M. Maraschek, R. M. McDermott, A. Mlynek, T. Pütterich, M. Rott, T. Vierle, E. Wolfrum, Q. Yu, I. Zammuto, and H. Zohm. First observation of edge localized modes mitigation with resonant and nonresonant magnetic perturbations in asdex upgrade. Phys. Rev. Lett., 106:225004, Jun 2011.

[5] Y. M. Jeon, J.-K. Park, S. W. Yoon, W. H. Ko, S. G. Lee, K. D. Lee, G. S. Yun, Y. U. Nam, W. C. Kim, Jong-Gu Kwak, K. S. Lee, H. K. Kim, and H. L. Yang. Suppression of edge localized modes in high-confinement kstar plasmas by nonaxisymmetric magnetic perturbations. Phys. Rev. Lett., 109:035004, Jul 2012.

[6] Y. Liang, H. R. Koslowski, P. R. Thomas, E. Nardon, B. Alper, P. Andrew, Y. Andrew, G. Arnoux, Y. Baranov, M. Becoulet, M. Beurskens, T. Biewer, M. Bigi, Kristel Crombé, E. De La Luna, P. de Vries, W. Fundamenski, S. Gerasimov, C. Giroud, M. P. Gryaznevich, N. Hawkes, S. Hotchin, D. Howell, S. Jachmich, V. Kiptily, L. Moreira, V. Parail, S. D. Pinches, E. Rachlew, and
O. Zimmermann. Active control of type-i edge-localized modes with $\mathrm{n}=1$ perturbation fields in the jet tokamak. Phys. Rev. Lett., 98(26):265004-1-265004-5, 2007.

[7] A. Kirk, E. Nardon, R. Akers, M. Bécoulet, G. De Temmerman, B. Dudson, B. Hnat, Y.Q. Liu, R. Martin, P. Tamain, D. Taylor, and the MAST team. Resonant magnetic perturbation experiments on mast using external and internal coils for elm control. Nuclear Fusion, 50(3):034008, 2010.

[8] J.M. Canik, R. Maingi, T.E. Evans, R.E. Bell, S.P. Gerhardt, H.W. Kugel, B.P. LeBlanc, J. Manickam, J.E. Menard, T.H. Osborne, J.-K. Park, S. Paul, P.B. Snyder, S.A. Sabbagh, E.A. Unterberg, and the NSTX team. Elm destabilization by externally applied non-axisymmetric magnetic perturbations in nstx. $\mathrm{Nu}$ clear Fusion, 50(3):034012, 2010.

[9] M.J. Schaffer, J.E. Menard, M.P. Aldan, J.M. Bialek, T.E. Evans, and R.A. Moyer. Study of in-vessel nonaxisymmetric elm suppression coil concepts for iter. Nuclear Fusion, 48(2):024004, 2008.

[10] H.R. Strauss, L. Sugiyama, G.Y. Park, C.S. Chang, $\mathrm{S}$. $\mathrm{Ku}$, and I. Joseph. Extended mhd simulation of resonant magnetic perturbations. Nuclear Fusion, 49(5):055025, 2009.

[11] E. Nardon, P. Tamain, M. Bécoulet, G. Huysmans, and F.L. Waelbroeck. Quasi-linear mhd modelling of h-mode plasma response to resonant magnetic perturbations. $\mathrm{Nu}$ clear Fusion, 50(3):034002, 2010.

[12] M. Bécoulet, F. Orain, P. Maget, N. Mellet, X. Garbet, E. Nardon, G. T. A. Huysmans, T. Casper, A. Loarte, P. Cahyna, A. Smolyakov, F. L. Waelbroeck, M. Schaffer, T. Evans, Y. Liang, O. Schmitz, M. Beurskens, V. Rozhansky, and E. Kaveeva. Screening of resonant magnetic perturbations by flows in tokamaks. Nuc. Fus., 52(5), MAY 2012. 5th International Workshop on Stochasticity in Fusion Plasmas (SFP), Julich, GERMANY, APR 11-14, 2011.

[13] N. M. Ferraro. Calculations of two-fluid linear response to non-axisymmetric fields in tokamaks. Physics of Plasmas, 19(5):056105, 2012.

[14] Yueqiang Liu, A. Kirk, and E. Nardon. Full toroidal plasma response to externally applied nonaxisymmetric magnetic fields. Physics of Plasmas, 17(12):122502, 2010.

[15] Q. Yu and S. Guenter. Plasma response to externally applied resonant magnetic perturbations. Nuclear Fusion, 51(7), JUL 2011.

[16] R Fitzpatrick. Bifurcated states of a rotating tokamak plasma in the presence of a static error-field. Physics of Plasmas, 5(9):3325-3341, 1998.

[17] G T A Huysmans, S Pamela, E van der Plas, and $P$ Ramet. Non-linear mhd simulations of edge localized modes (elms). Plasma Physics and Controlled Fusion, 51(12):124012, 2009.

[18] HR Strauss. Reduced mhd in nearly potential magnetic fields. Journal of Plasma Physics, 57(Part 1):83-87, JAN 1997.

[19] R. D. Hazeltine, M. Kotschenreuther, and P. J. Morrison. A four-field model for tokamak plasma dynamics. Physics of Fluids, 28(8):2466-2477, 1985.

[20] TA Gianakon, SE Kruger, and CC Hegna. Heuristic closures for numerical simulations of neoclassical tearing 
modes. Physics of Plasmas, 9(2):536-547, 2002.

[21] C.E. Kessel. Bootstrap current in a tokamak. Nuclear Fusion, 34(9):1221, 1994.

[22] M. Bécoulet, E. Nardon, G. Huysmans, W. Zwingmann, P. Thomas, M. Lipa, R. Moyer, T. Evans, V. Chuyanov, Y. Gribov, A. Polevoi, G. Vayakis, G. Federici, G. Saibene, A. Portone, A. Loarte, C. Doebert, C. Gimblett, J. Hastie, and V. Parail. Numerical study of the resonant magnetic perturbations for type i edge localized modes control in iter. Nuclear Fusion, 48(2):024003, 2008.

[23] S. Nishimura, S. Toda, M. Yagi, and Y. Narushima. Nonlinear stability of magnetic islands in a rotating helical plasma. Physics of Plasmas, 19(12), DEC 2012.

[24] Y. Andrew, N. C. Hawkes, T. Biewer, K. Crombe, D. Keeling, E. De La Luna, C. Giroud, A. Korotkov, A. Meigs, A. Murari, I. Nunes, R. Sartori, T. Tala, and JET-EFDA Contributors. Evolution of the radial electric field in a jet h-mode plasma. EPL, 83(1), 2008.

[25] F. Orain, M. Bécoulet, G. T. A. Huysmans, G. DifPradalier, V. Grandgirard, G. Latu, P. Maget, N. Mellet, E. Nardon, C. Passeron, and A. Ratnani. Interaction of resonant magnetic perturbations with flows in toroidal geometry. 2012. 39th European Physical Society Conference on Plasma Physics and 16th International Congress on Plasma Physics Stockholm, Sweden, 2-6 July 2012.

[26] V. A. Izzo and I. Joseph. Rmp enhanced transport and rotational screening in simulations of diii-d plasmas. $\mathrm{Nu}$ clear Fusion, 48(11), NOV 2008.

[27] RA Moyer, TE Evans, TH Osborne, PR Thomas, M Bécoulet, J Harris, KH Finken, JA Boedo, EJ Doyle, ME Fenstermacher, P Gohil, RJ Groebner, M Groth, GL Jackson, RJ La Haye, CJ Lasnier, AW Leonard, GR McKee, H Reimerdes, TL Rhodes, DL Rudakov, MJ Schaffer, PB Snyder, MR Wade, G Wang, JG Watkins, WP West, and L Zeng. Edge localized mode control with an edge resonant magnetic perturbation. Physics of Plasmas, 12(5), MAY 2005.

[28] Martin F. Heyn, Ivan B. Ivanov, Sergei V. Kasilov, Winfried Kernbichler, Ilon Joseph, Richard A. Moyer, and Alexey M. Runov. Kinetic estimate of the shielding of resonant magnetic field perturbations by the plasma in diii-d. Nuclear Fusion, 48(2):024005, 2008.

[29] F.L. Waelbroeck, I. Joseph, E. Nardon, M. Bécoulet, and R. Fitzpatrick. Role of singular layers in the plasma response to resonant magnetic perturbations. Nuclear Fusion, 52(7):074004, 2012.

[30] Yueqiang Liu, M. S. Chu, Y. In, and M. Okabayashi. Resonant field amplification with feedback-stabilized regime in current driven resistive wall mode. Physics of Plasmas, 010, Volume $=17$, Number $=7$, Month $=$ JUL

[31] I. Joseph, T.E. Evans, A.M. Runov, M.E. Fenstermacher, M. Groth, S.V. Kasilov, C.J. Lasnier, R.A. Moyer, G.D. Porter, M.J. Schaffer, R. Schneider, and J.G. Watkins. Calculation of stochastic thermal transport due to resonant magnetic perturbations in DIII-D. Nuclear Fusion, 48(4):045009, 2008.

[32] T E Evans, R K W Roeder, J A Carter, B I Rapoport, M E Fenstermacher, and C J Lasnier. Experimental signatures of homoclinic tangles in poloidally diverted tokamaks. Journal of Physics: Conference Series, 7:174-190, 2005.

[33] A. Kirk, J. Harrison, Yueqiang Liu, E. Nardon, I. T. Chapman, and P. Denner. Observation of lobes near the $x$ point in resonant magnetic perturbation experiments on mast. Phys. Rev. Lett., 108:255003, Jun 2012.

[34] I. T. Chapman, A. Kirk, S. Saarelma, J. R. Harrison, R. Scannell, and MAST Team. Towards understanding elm mitigation: the effect of axisymmetric lobe structures near the x-point on elm stability. Nuclear Fusion, 52(12), DEC 2012.

[35] P. Cahyna, M. Peterka, A. Kirk, A. Thornton, J. Harrison, D. Muir, and R. Panek. Strike point splitting induced by the application of magnetic perturbations on \{MAST\}. Journal of Nuclear Materials, (0):-, 2013.

[36] T.E. Evans, I. Joseph, R.A. Moyer, M.E. Fenstermacher, C.J. Lasnier, and L.W. Yan. Experimental and numerical studies of separatrix splitting and magnetic footprints in DIII-D. Journal of Nuclear Materials, 363-365:570 - 574, 2007. Plasma-Surface Interactions-17.

[37] O Schmitz, T E Evans, M E Fenstermacher, H Frerichs, M W Jakubowski, M J Schaffer, A Wingen, W P West, N H Brooks, K H Burrell, J S deGrassie, Y Feng, K H Finken, P Gohil, M Groth, I Joseph, C J Lasnier, M Lehnen, A W Leonard, S Mordijck, R A Moyer, A Nicolai, T H Osborne, D Reiter, U Samm, K H Spatschek, H Stoschus, B Unterberg, E A Unterberg, J G Watkins, R Wolf, and the DIII-D and TEXTOR Teams. Aspects of three dimensional transport for ELM control experiments in ITER-similar shape plasmas at low collisionality in DIII-D. Plasma Physics and Controlled Fusion, 50(12):124029, 2008.

[38] E. Nardon, P. Cahyna, S. Devaux, A. Kirk, A. Alfier, E. De La Luna, G. de Temmerman, P. Denner, T. Eich, T. Gerbaud, D. Harting, S. Jachmich, H.R. Koslowski, Y. Liang, and Y. Sun. Strike-point splitting induced by external magnetic perturbations: observations on JET and MAST and associated modelling. Journal of Nuclear Materials, 415(1, Supplement):S914-S917, 2011.

[39] P. Cahyna, E. Nardon, and JET EFDA contributors. Model for screening of resonant magnetic perturbations by plasma in a realistic tokamak geometry and its impact on divertor strike points. Journal of Nuclear Materials, 415(1, Supplement):S927-S931, 2011.

[40] P. Cahyna, Y.Q. Liu, E. Nardon, A. Kirk, M. Peterka, J.R. Harrison, A. Thornton, I. Chapman, R. Panek, O. Schmitz, and the MAST team. Modelling of plasma response to resonant magnetic perturbations and its influence on divertor strike points. In IAEA Fusion Energy Conference 2012 (Proc. 24th IAEA FEC San Diego, 2012), pages TH/P4-27, (Vienna: IAEA), 2013.

[41] O. Schmitz, M. Becoulet, P. Cahyna, T.E. Evans, Y. Feng, H. Frerichs, A. Kischner, A. Kukushkin, R. Laengner, T. Lunt, A. Loarte, R. Pitts, D. Reiser, D. Reiter, G. Saibene, and U. Samm. Modeling of divertor particle and heat loads during application of resonant magnetic perturbation fields for $\{\mathrm{ELM}\}$ control in \{ITER\}. Journal of Nuclear Materials, (0):-, 2013.

[42] H. Frerichs, D. Reiter, O. Schmitz, P. Cahyna, T. E. Evans, Y. Feng, and E. Nardon. Impact of screening of resonant magnetic perturbations in three dimensional edge plasma transport simulations for DIII-D. Physics of Plasmas, 19(5):052507, 2012.

[43] A. Kirk, Yueqiang Liu, E. Nardon, P. Tamain, P. Cahyna, I. Chapman, P. Denner, H. Meyer, S. Mordijck, D. Temple, and MAST Team. Magnetic perturbation experiments on mast l-and h-mode plasmas using internal coils. Plasma Physics and Controlled Fusion, 53(6), JUN 2011. 
[44] A. Kirk, I. T. Chapman, J. Harrison, Yueqiang Liu, E. Nardon, S. Saarelma, R. Scannell, A. J. Thornton, and MAST Team. Effect of resonant magnetic perturbations with toroidal mode numbers of 4 and 6 on edge-localized modes in single null h-mode plasmas in mast. Plasma Physics and Controlled Fusion, 55(1), JAN 2013.

[45] J.D. Callen, A.J. Cole, and C.C.Hegna. Nuclear Fusion,
49:085021, 2009

[46] M. Bécoulet, G. Huysmans, X. Garbet, E. Nardon, D. Howell, A. Garofalo, M. Schaffer, T. Evans, K. Shaing, A. Cole, J.-K. Park, and P. Cahyna Nuclear Fusion 49:085011, 2009 